\title{
Vitamin D receptor gene polymorphisms and susceptibility to urolithiasis: a meta- regression and meta-analysis
}

\author{
Danyal Imani', Bahman Razi ${ }^{1}$, Arezou Khosrojerdi ${ }^{3}$, Kaivan Lorian ${ }^{4}$, Morteza Motallebnezhad, ${ }^{5,6}$, \\ Ramazan Rezaei ${ }^{7}$ and Saeed Aslani ${ }^{8^{*}}$
}

\begin{abstract}
Background: The currently available data with respect to the association between vitamin D receptor (VDR) gene polymorphism and risk to urolithiasis are inconclusive and inconsistent. Hence, an exhaustive meta-analysis can solve the discrepancies and provide a hint for upcoming investigations. Herein, a meta-analysis was carried out to attain a conclusive estimate of the association between VDR gene single nucleotide polymorphisms (SNPS) and urolithiasis risk.

Methods: The major databases, including ISI Web of science, Scopus, and PubMed/MEDLINE were searched systematically from until June 2020 to retrieve all relevant studies. Association between VDR gene polymorphisms, including Fokl (rs2228570), Taql (rs731236), Bsml (rs1544410), and Apal (rs7975232), and urolithiasis risk was evaluated using pooled odds ratio (OR) and their corresponding 95\% confidence interval (Cl). Additionally, to seek for the potential source of heterogeneity, meta-regression analyses were exerted.

Results: Literature search led to finally finding of 33 studies evaluating the VDR gene SNPs and urolithiasis risk. It was observed that none of the four SNPs were significantly associated with urolithiasis predisposition. However, subgroup analysis confirmed higher risk of urolithiasis in East-Asian and Caucasian population with Apal and Taql gene polymorphism. The analyses of sensitivity acknowledged the results stability.

Conclusion: Although this meta-analysis did not support the association of Fokl, Taql, Bsml, and Apal in the overall polled analysis, it suggests that Apal and Taq/ SNPs is associated with increased risk of urolithiasis in East-Asian and Caucasians populations.
\end{abstract}

Keywords: Vitamin D receptor, Urolithiasis, Meta-analysis, Polymorphism

\section{Background}

Urolithiasis is known as one of the prevalent diseases among urological disorders that has been associated with many complicated factors [1]. Urolithiasis is characterized by a high recurrence incidence, and its prevalence rate is $4-20 \%$ in developed countries, and the disease

\footnotetext{
* Correspondence: s-aslani@razi.tums.ac.ir

${ }^{8}$ Department of Immunology, School of Medicine, Tehran University of Medical Sciences, Tehran, Iran

Full list of author information is available at the end of the article
}

incidence continues to increase [2]. It is a multifactorial disorder, resulting from environmental influences, metabolic defects and genetic factors [3]. Numerous investigations recognized the importance of genes in this disorder.

Studies have shown that several genetic factors including single nucleotide polymorphisms (SNPs) in osteopontin (OPN), progestin and adiporeceptor 6 (PAQR6), calcium-sensing receptor (CaSR), and vitamin D receptor (VDR) are correlated with the risk of urinary calcium 
stone formation [4-7]. In spite of attribution of a genetic background in susceptibility to urolithiasis, little has been identified with respect to the relevant genetic loci for the disease. Two genome-wide association studies (GWASs) recognized four risk susceptibility genes, including CLDN14 in Europeans and Japanese $[8,9]$, INMT-FAM188B-AQP1, RGS14-SLC34A1-PFN3-F12, and $D G K H$ in Japanese [9]. That notwithstanding, these studies suggested further studies was needed to identify more risk loci as well as to recognize the molecular mechanisms attributed to the urinary calculi.

Broadly speaking, complex interactions of genetics and environmental factors, such as water intake, diet, urine $\mathrm{pH}$, and infections have been associated with the etiopathogenesis of urolithiasis [10]. The underlying mechanisms of the development of calcium-containing stones, which are the most common type of kidney and bladder stones, have not fully been divulged [11]. Nowadays, the possibility of both free and fixed stones development has been suggested. The widely accepted explanation of the development of such stones relies on the increased solubility of the lithogenic elements in the urine [11]. Furthermore, it has been contemplated that the deposition of initial crystals occurs in the lumens of renal tubules $[12,13]$. However, recent observations imply that a development of Randall plaques in the renal papilla is the initial trigger of stone formation [14]. Such plaques are developed when calcium phosphate crystals are deposited in the basement of the thin loops of Henle and then extend into the urothelium. Calcium oxalate stones, which are responsible for almost $80 \%$ of all urinary stones, are developed after formation of calcium phosphate crystals. In fact, the binding of more calcium oxalate as well as matrix molecules present in the urine to the Randall plaques accelerates the formation of calcium oxalate stones [15].

Recent studies have demonstrated that receiving vitamin $\mathrm{D}$ supplements maybe put the individual at risk of developing kidney stones disease [16]. Moreover, vitamin D has an important role in calcium metabolism, such as absorption of calcium from intestine and its reabsorption in the kidneys. it through increasing the serum calcium levels could enhance the risk of urinary stone formation [17]. Vitamin D functions are dependent on the expression and nuclear activation of VDR [18]. Therefore, any alteration in the VDR may change the calcium metabolism, thus alter the urolithiasis risk. Taken together, studies have recommended that VDR play an essential role in the pathogenesis of urolithiasis [19].

The human $V D R$ gene is placed on the chromosome 12q12-q14 that harbors more than 200 SNPs, among which FokI (or rs2228570), TaqI (or rs731236), BsmI (or rs1544410), and ApaI (or rs7975232) polymorphisms have been extensively investigated. $V D R$ gene has at least five promoter regions, six untranslated exons, and eight protein-coding exons, which are alternatively spliced into BsmI, FokI, ApaI, and TaqI [20].. BsmI and ApaI are placed on the 9th intron of the $3^{\prime}$ terminal, TaqI is located on the 9th exon of the $3^{\prime}$ terminal, and FokI is established on the promoter of the $5^{\prime}$ terminal. Studies have reported that BsmI and TaqI SNPs are not involved in altering the protein structure of VDR; however, they have been suggested to play a role in the translation efficiency and stability of the corresponding mRNA [21]. Numerous studies have indicated the association of polymorphisms in the $V D R$ gene with several human diseases [22, 23].

A series of studies investigated the association between these polymorphisms of $V D R$ gene and the risk of urolithiasis, but the findings have been conflicting [24-50]. The inconsistent results were possibly because of clinical heterogeneity, small sample sizes, and low statistical power. In addition, previous meta-analyses [51-53] appeared to be out of date due to the availability of new data [45-50]. Therefore, we performed the most up to date meta-analysis with the aim of obtaining more accurate and updated results.

\section{Methods}

This study was performed in a stepwise process in accordance with the guidelines of the 2009 Preferred Reporting Items for Systematic Reviews and Metaanalyses (PRISMA) statement [54]. Besides, the current project does not contain any studies with human participants or animals performed by any of the authors. Registration in the International Prospective Register of Systematic Reviews (PROSPERO) was carried out.

\section{Literature identification}

A detailed systematic search was performed to identify candidate studies evaluating the associations between $V D R$ gene polymorphisms and urolithiasis susceptibility (prior to June 2020). Three electronic databases, including Web of Science, MEDLINE, and Scopus were searched and for all of them, following combination of key words were used: ("urolithiasis" or "Kidney stone disease") AND ("VDR" OR "vitamin D receptor") AND ("polymorphisms" OR "SNP" OR "variation" OR "mutation"). Cross references within both original and review publications were done for additional pertinent studies. Original data were collected from English language and human population studies.

\section{Inclusion/exclusion criteria}

Studies included in quantitative analysis if met the following inclusion criteria: a) studies concerning the association between $V D R$ gene polymorphisms and 
urolithiasis risk; b) Studies with case-control design; c) studies reporting sufficient data of genotype or allele frequency in order to calculate odds ratios (ORs) and 95\% confidence intervals (CIs). On the other hand, duplicate data, case report, book chapter, review, letter, and abstracts were excluded.

\section{Data extraction}

All required data were extracted conforming to the standardized extraction checklist for the following data: the first author's name, journal and year of publication, country of origin, ethnicity, number of subjects in the case and control groups, mean or range of age, genotyping method, genotype counts in the case and control group. The extracted items were compared and any possible discrepancies were resolved by consensus.

\section{Quality assessment}

Methodological quality of eligible studies was evaluated by Newcastle-Ottawa Scale (NOS), a validated scale for non-randomized studies in meta-analysis. This scale consists of 3 parts with a total of 9 items. In this regards, studies with scores $0-3,4-6$ or 7-9 were of low, moderate, or high-quality, respectively [55].

\section{Statistical analysis}

For evaluating the distribution of the genotype frequencies to see if it is deviated from Hardy-Weinberg equilibrium (HWE) in the control group, the $\mathrm{x}^{2}$-test was employed [56]. The quality of association between $V D R$ gene SNPs and urolithiasis risk was evaluated by the pooled OR and its corresponding 95\% CI. Five different comparison model for FokI, TaqI, BsmI, and ApaI SNPs were as follow: dominant model, recessive model, allelic model, homozygote contrast, and heterozygote contrast. Presence of heterogeneity between included studies was estimated by Cochran's Q-statistic ( $P$ value $<0.10$ was considered as statistically significant). Besides, to report quantitative heterogeneity, the I-squared (I2) test was used. The fixed-effected model was used if PQ statistic> 0.10 or I2 was $<5 \%$; otherwise, the random-effected model was applied $[57,58]$. We assessed the predefined sources of heterogeneity among included studies by subgroup analysis and meta-regression analysis based on year of population, and genotyping method. The stability of our results was measured by sensitivity analysis. Additionally, sensitivity analysis was conducted in the presence of heterogeneity. Moreover, Begg's test, Egger's regression test and visual examination of the funnel plot were applied to measure publication bias $(P$ value $<0.05$ was considered as statistically significant) [59]. The data analyses were carried out using STATA (version 14.0; Stata Corporation, College Station, TX) and SPSS (version 23.0; SPSS, Inc. Chicago, IL).

\section{Results}

\section{Specifications of the included studies}

The exact process of literature searches and study selection is depicted in the Fig. 1. Early literature search eventuated in identification of 207 records, 33 of which met the final inclusion criteria and included in quantitative analysis. Among 33 eligible studies, 20 studies investigated the FokI SNP, 22 studies TaqI SNP, 14 studies BsmI SNP and 16 studies ApaI SNP. The studies were published between 1999 and 2020 and had an overall good methodological quality with NOS scores ranging from 5 to 8 . Polymerase chain reaction-restriction fragment length polymorphism (PCR-RFLP) and Taq-Man were used by majority of included studies as genotyping method. Tables 1 and 2 summarized the characteristics of the included studies.

\section{Quantitative synthesis}

As the reference categories for comparing, the FF genotype for FokI SNP, TT genotype for TaqI SNP, BB genotype for BsmI SNP, and AA genotype for ApaI were used.

\section{Association of Fokl polymorphism and urolithiasis risk} Twenty studies, including 3114 urolithiasis patients and 3174 controls, evaluated the FokI polymorphism. Of which, 14 studies were conducted in Asian countries $[26,28,34,35,37,40-42,44,50,60-63]$ and 6 studies were in European countries [31-33, 48, 64, 65]. Overall, no significant association was detected between FokI SNP and urolithiasis risk under all five genetic models. Besides, the findings of subgroup analysis reject any association between FokI SNP and risk of urolithiasis in East-Asians and Caucasians.

\section{Association of Taql polymorphism and urolithiasis risk}

Twenty-two case-control studies with 4188 cases and 3955 controls met inclusion criteria for evaluating the association between TaqI SNP and urolithiasis risk. Among them, ten studies were performed in Asian population $[28,40-42,44,60-63,66]$ and eleven studies were in European population [29, 31, 36, 38, 39, 45, 46, $48,65,67]$ and one study in the USA [24]. The pooled results did not indicate significant association between TaqI SNP and urolithiasis risk except in tt vs. TT model $(\mathrm{OR}=1.27,95 \% \mathrm{CI}=1.01-1.59, P=0.04)$, also subgroup analysis revealed that the tt genotype was associated with urolithiasis risk in Caucasians when compared with the TT genotype [tt vs. TT $(\mathrm{OR}=1.30,95 \% \mathrm{CI}=1.021-1.65$, $P=0.03)$ ], Fig. 2 .

\section{Association of Bsml polymorphism and urolithiasis risk}

Fourteen eligible publications with 3065 cases and 2915 controls were included and evaluated the association 


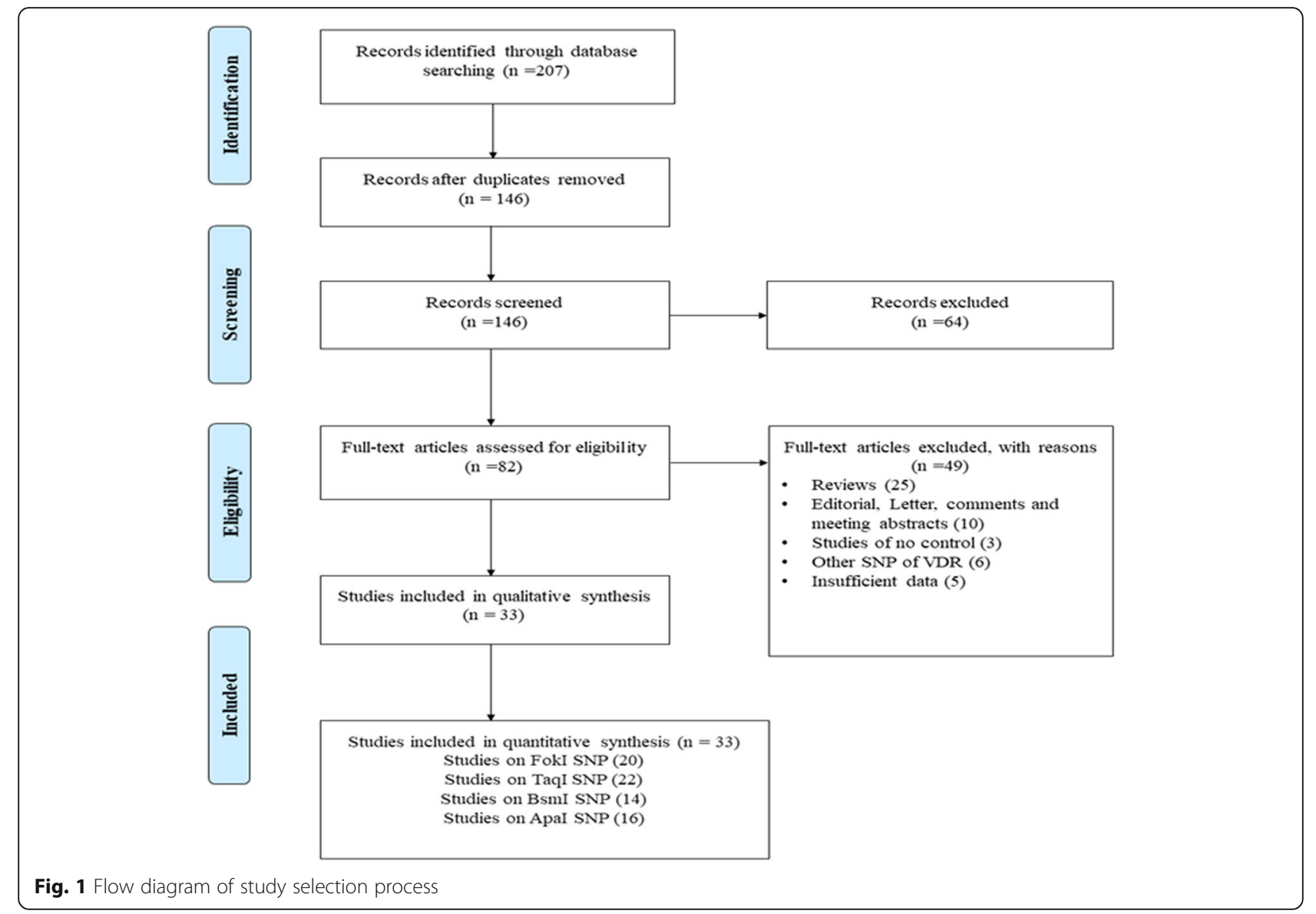

between BsmI polymorphism and urolithiasis risk. Among 14 studies, only five publications were carried out in Asia [27, 44, 62, 66, 68] and nine studies were in Europe [25, 29, 31-33, 36, 38, 46, 48, 65]. The statistical analysis demonstrated that there was no significant association between BsmI SNP and urolithiasis risk under any genetic models in both the overall population and the subgroup analysis.

\section{Association of Apal polymorphism and urolithiasis risk}

A total of 16 publications containing 2950 cases and 3065 controls were recognized eligible for evaluating the association between ApaI SNP and urolithiasis risk. Of which, eight studies were performed in Asians [27, 28, $40,44,61,66,68]$ and eight studies were in Europeans $[29,33,36,38,41,45,46,48,64]$. The pooled results revealed a marginal significant association between ApaI SNP and urolithiasis risk under recessive model $(\mathrm{OR}=$ $1.14,95 \% \mathrm{CI}=1.01-1.29, p=0.03)$, allelic model (OR = $1.09,95 \% \mathrm{CI}=1-1.18, P=0.05)$, and aa vs. AA model $(\mathrm{OR}=1.21,95 \% \mathrm{CI}=1-1.47, P=0.05)$. Additionally, the results of subgroup analysis indicated a positive significant association in East-Asians across recessive model $(\mathrm{OR}=1.20,95 \% \mathrm{CI}=1.05-1.37, P<0.001)$, allelic model
$(\mathrm{OR}=1.15,95 \% \mathrm{CI}=1.05-1.26, P<0.001)$, and aa vs. AA model $(\mathrm{OR}=1.40,95 \% \mathrm{CI}=1.12-1.75, P<0.001)$ but not Caucasians. The results of pooled ORs, heterogeneity tests, and publication bias tests for different analysis models are shown in Table 3.

\section{Evaluation of the heterogeneity and publication bias}

The results of publication bias test indicated that there was no evidence of publication bias for overall population and subgroup analysis of all FokI, TaqI, BsmI, and ApaI SNPs. Additionally, the shape of the funnel plot confirmed absence of publication bias. No heterogeneity in both the overall and subgroup analyses was detected except for FokI polymorphism (Fig. 3, Table 3).

\section{Sensitivity analysis}

Sensitivity analysis is an effective test to evaluate the influence of individual study on the pooled results. In the sensitivity analysis, each eligible study was sequentially removed to assess whether the individual data influence the pooled ORs. In this meta-analysis, the pooled results did not significantly affect by any single study in the dominant model for FokI, TaqI, BsmI and ApaI SNPs 
Table 1 Characteristics of studies included in meta-analysis of overall Urolithiasis

\begin{tabular}{|c|c|c|c|c|c|c|c|c|}
\hline Study author & Year & Country & Ethnicity & $\begin{array}{l}\text { Sex } \\
\text { cases/controls }\end{array}$ & Total cases/controls & $\begin{array}{l}\text { Age } \\
\text { case/control (Mean) }\end{array}$ & Genotyping method & $\begin{array}{l}\text { Quality } \\
\text { score }\end{array}$ \\
\hline \multicolumn{9}{|l|}{ Fokl (rs2228570) } \\
\hline Chen et al. (b) & 2001 & China & Asian & $\begin{array}{l}M=101 / 42 \\
F=45 / 48\end{array}$ & $146 / 90$ & $44.2 / 55.5$ & PCR-RFLP & 6 \\
\hline Shaogang et al. & 2003 & China & Asian & $\begin{array}{l}M=89 / 58 \\
F=61 / 22\end{array}$ & $150 / 80$ & $43.6 \pm 16 / 49 \pm 19.6$ & PCR-RFLP & 6 \\
\hline Rendina et al. & 2004 & Italy & European & $\begin{array}{l}M=94 / 72 \\
F=65 / 52\end{array}$ & 159 / 124 & $43 \pm 10.8 / 41.9 \pm 10.4$ & PCR-RFLP & 7 \\
\hline Mossetti et al. & 2004 & Italy & European & $\begin{array}{l}M=66 / 73 \\
F=44 / 54\end{array}$ & $110 / 127$ & $41.3 \pm 13.5 / 41.06 \pm 13.9$ & PCR-RFLP & 6 \\
\hline Relan et al. & 2004 & India & Asian & $\begin{array}{l}M=105 / 76 \\
F=45 / 24\end{array}$ & $150 / 100$ & $39.3 \pm 1.1 / 43.2 \pm 2.05$ & PCR-RFLP & 7 \\
\hline Bid et al. (a) & 2005 & India & Asian & $\begin{array}{l}M=N R \\
F=N R\end{array}$ & $113 / 132$ & $21-72 / 22-58$ & PCR-RFLP & 6 \\
\hline Bid et al. (b) & 2005 & India & Asian & $\begin{array}{l}M=N R \\
F=N R\end{array}$ & $50 / 60$ & $2-14 / 4-16$ & PCR-RFLP & 5 \\
\hline Liu et al. & 2007 & China & Asian & $\begin{array}{l}M=161 / 159 \\
F=74 / 72\end{array}$ & $235 / 231$ & $50.1 \pm 12.3 / 51.7 \pm 11.1$ & PCR-RFLP & 8 \\
\hline Seo et al. & 2009 & Korea & Asian & $\begin{array}{l}M=93 / 220 \\
F=185 / 313\end{array}$ & $278 / 533$ & 49.9 / 40.1 & PCR-RFLP & 8 \\
\hline Mittal et al. & 2010 & India & Asian & $\begin{array}{l}M=N R \\
F=N R\end{array}$ & $125 / 150$ & $40 \pm 11.5 / 41.5 \pm 10.5$ & PCR-RFLP & 7 \\
\hline Basiri et al. & 2012 & Iran & Asian & $\begin{array}{l}M=N R \\
F=N R\end{array}$ & $102 / 106$ & $43.4 \pm 6.9 / 38.4 \pm 6.9$ & SSP-PCR & 6 \\
\hline Kaysar et al. & 2012 & China & Asian & $\begin{array}{l}M=N R \\
F=N R\end{array}$ & $74 / 103$ & NR & PCR-RFLP & 5 \\
\hline Wang et al. & 2012 & China & Asian & $\begin{array}{l}M=279 / 263 \\
F=185 / 187\end{array}$ & $464 / 450$ & $50.01 \pm 10.19 / 50.45 \pm 11.22$ & PCR-RFLP & 8 \\
\hline Guha et al. & 2015 & India & Asian & $\begin{array}{l}M=133 / 112 \\
F=67 / 78\end{array}$ & $200 / 200$ & $39.93 \pm 11 / 38.13 \pm 10$ & PCR & 7 \\
\hline Cakir et al. & 2016 & Turkey & European & $\begin{array}{l}M=65 / 52 \\
F=33 / 18\end{array}$ & $98 / 70$ & $47.2 \pm 16.3 / 42.6 \pm 13.5$ & PCR-RFLP & 6 \\
\hline Ergon et al. & 2017 & Turkey & European & $\begin{array}{l}M=N R \\
F=N R\end{array}$ & $27 / 13$ & $7.12 \pm 2.64 / 6.92 \pm 2.48$ & Tag-man & 5 \\
\hline Subasi et al. & 2017 & Turkey & European & $\begin{array}{l}M=26 / 22 \\
F=26 / 29\end{array}$ & $52 / 51$ & $9.8 \pm 3.5 / 10.3 \pm 3.7$ & SNaPshot & 5 \\
\hline Li et al. & 2018 & China & Asian & $\begin{array}{l}M=100 / 60 \\
F=100 / 60\end{array}$ & $200 / 120$ & $35.88 \pm 14.2 / 36.16 \pm 15.20$ & PCR & 7 \\
\hline Huang et al. & 2019 & China & Asian & $\begin{array}{l}M=N R \\
F=N R\end{array}$ & $130 / 224$ & $4.55 \pm 3.19 / 5.02 \pm 3.50$ & PCR-RFLP & 7 \\
\hline Amar et al. & 2019 & Pakistani & Asian & $\begin{array}{l}M=N R \\
F=N R\end{array}$ & $235 / 243$ & NR & PCR-RFLP & 7 \\
\hline \multicolumn{9}{|l|}{ Taql (rs731236) } \\
\hline Jackman et al. & 1999 & USA & American & $\begin{array}{l}M=N R \\
F=N R\end{array}$ & $17 / 37$ & NR & PCR-RFLP & 5 \\
\hline Nishijima et al. & 2002 & Japan & Asian & $\begin{array}{l}M=N R \\
F=N R\end{array}$ & $83 / 83$ & $51.8 \pm 15.6 / 54.4 \pm 13.1$ & PCR-RFLP & 5 \\
\hline Ozkaya et al. & 2003 & Turkey & European & $\begin{array}{l}M=26 / 47 \\
F=38 / 43\end{array}$ & $64 / 90$ & $6.7 \pm 3.5 / 7.2 \pm 2.3$ & PCR-RFLP & 5 \\
\hline Mossetti et al. & 2003 & Italy & European & $\begin{array}{l}M=N R \\
F=N R\end{array}$ & 220/114 & $40.87 \pm 14.95 / 40.37 \pm 14.07$ & PCR-RFLP & 7 \\
\hline Shaogang et al. & 2003 & China & Asian & $\begin{array}{l}M=89 / 58 \\
F=61 / 22\end{array}$ & $150 / 80$ & $43.6 \pm 16 / 49 \pm 19.6$ & PCR-RFLP & 6 \\
\hline
\end{tabular}


Table 1 Characteristics of studies included in meta-analysis of overall Urolithiasis (Continued)

\begin{tabular}{|c|c|c|c|c|c|c|c|c|}
\hline Study author & Year & Country & Ethnicity & $\begin{array}{l}\text { Sex } \\
\text { cases/controls }\end{array}$ & Total cases/controls & $\begin{array}{l}\text { Age } \\
\text { case/control (Mean) }\end{array}$ & Genotyping method & $\begin{array}{l}\text { Quality } \\
\text { score }\end{array}$ \\
\hline Mossetti et al. & 2004 & Italy & European & $\begin{array}{l}M=66 / 73 \\
F=44 / 54\end{array}$ & $110 / 127$ & $41.3 \pm 13.5 / 41.06 \pm 13.9$ & PCR-RFLP & 6 \\
\hline Gunes et al. & 2006 & Turkey & European & $\begin{array}{l}M=67 / 73 \\
F=43 / 77\end{array}$ & $110 / 150$ & $49.22 \pm 1.33 / 48.15 \pm 1.62$ & PCR-RFLP & 7 \\
\hline Sayan et al. & 2007 & Turkey & European & $\begin{array}{l}M=65 / 25 \\
F=15 / 15\end{array}$ & $80 / 40$ & $10.9 \pm 0.6 / 10.5 \pm 0.6$ & PCR-RFLP & 5 \\
\hline Moyano et al. & 2007 & Spain & European & $\begin{array}{l}M=22 / 9 \\
F=29 / 12\end{array}$ & $51 / 21$ & $45.5 \pm 13.5 / 48.6 \pm 15.4$ & PCR-RFLP & 5 \\
\hline Seo et al. & 2009 & Korea & Asian & $\begin{array}{l}M=93 / 220 \\
F=185 / 313\end{array}$ & $278 / 533$ & 49.9 / 40.1 & PCR-RFLP & 8 \\
\hline Mittal et al. & 2010 & India & Asian & $\begin{array}{l}M=N R \\
F=N R\end{array}$ & $125 / 150$ & $40 \pm 11.5 / 41.5 \pm 10.5$ & PCR-RFLP & 7 \\
\hline Basiri et al. & 2012 & Iran & Asian & $\begin{array}{l}M=N R \\
F=N R\end{array}$ & $102 / 106$ & $43.4 \pm 6.9 / 38.4 \pm 6.9$ & SSP-PCR & 6 \\
\hline Wang et al. & 2012 & China & Asian & $\begin{array}{l}M=279 / 263 \\
F=185 / 187\end{array}$ & $464 / 450$ & $50.01 \pm 10.19 / 50.45 \pm 11.22$ & PCR-RFLP & 8 \\
\hline Aykan et al. & 2015 & Turkey & European & $\begin{array}{l}M=100 / 87 \\
F=64 / 80\end{array}$ & 164 / 167 & $24-58 / 38-54$ & PCR-RFLP & 7 \\
\hline Guha et al. & 2015 & India & Asian & $\begin{array}{l}M=133 / 112 \\
F=67 / 78\end{array}$ & $200 / 200$ & $39.93 \pm 11 / 38.13 \pm 10$ & PCR & 7 \\
\hline Rendina et al. & 2016 & Italy & European & $\begin{array}{l}M=N R \\
F=N R\end{array}$ & $372 / 88$ & $41.2 \pm 13.3 / 40.8 \pm 14.1$ & PCR-RFLP & 7 \\
\hline Cakir et al. & 2016 & Turkey & European & $\begin{array}{l}M=65 / 52 \\
F=33 / 18\end{array}$ & $98 / 70$ & $47.2 \pm 16.3 / 42.6 \pm 13.5$ & PCR-RFLP & 6 \\
\hline Goknar et al. & 2016 & Turkey & European & $\begin{array}{l}M=N R \\
F=N R\end{array}$ & $78 / 60$ & $6.94 \pm 3.8 / 7.5 \pm 3.2$ & PCR-RFLP & 6 \\
\hline Subasi et al. & 2017 & Turkey & European & $\begin{array}{l}M=26 / 22 \\
F=26 / 29\end{array}$ & $52 / 51$ & $9.8 \pm 3.5 / 10.3 \pm 3.7$ & SNaPshot & 5 \\
\hline Li et al. & 2018 & China & Asian & $\begin{array}{l}M=100 / 60 \\
F=100 / 60\end{array}$ & $200 / 120$ & $35.88 \pm 14.2 / 36.16 \pm 15.20$ & PCR & 7 \\
\hline Yang et al. & 2019 & China & Asian & $\begin{array}{l}M=627 / 614 \\
F=316 / 361\end{array}$ & $943 / 975$ & $51.2 \pm 14.13 / 54.33 \pm 18.11$ & iMLDR & 8 \\
\hline Amar et al. & 2019 & Pakistani & Asian & $\begin{array}{l}M=N R \\
F=N R\end{array}$ & $227 / 243$ & NR & PCR-RFLP & 7 \\
\hline \multicolumn{9}{|l|}{ Bsml (rs1544410) } \\
\hline Ruggiero et al. & 1999 & Italy & European & $\begin{array}{l}M=18 / N R \\
F=9 / N R\end{array}$ & $27 / 150$ & NR & PCR-RFLP & 6 \\
\hline Chen et al. (a) & 2001 & China & Asian & $\begin{array}{l}M=94 / 55 \\
F=30 / 35\end{array}$ & $124 / 90$ & $44.1 \pm 11.5 / 53 \pm 10.1$ & PCR-RFLP & 6 \\
\hline Ozkaya et al. & 2003 & turkey & European & $\begin{array}{l}M=26 / 47 \\
F=38 / 43\end{array}$ & $64 / 90$ & $6.7 \pm 3.5 / 7.2 \pm 2.3$ & PCR-RFLP & 5 \\
\hline Rendina et al. & 2004 & Italy & European & $\begin{array}{l}M=94 / 72 \\
F=65 / 52\end{array}$ & $159 / 124$ & $43 \pm 10.8 / 41.9 \pm 10.4$ & PCR-RFLP & 7 \\
\hline Mossetti et al. & 2004 & Italy & European & $\begin{array}{l}M=66 / 73 \\
F=44 / 54\end{array}$ & $110 / 127$ & $41.3 \pm 13.5 / 41.06 \pm 13.9$ & PCR-RFLP & 6 \\
\hline Relan et al. & 2004 & India & Asia & $\begin{array}{l}M=105 / 76 \\
F=45 / 24\end{array}$ & $150 / 100$ & $39.3 \pm 1.1 / 43.2 \pm 2.05$ & PCR-RFLP & 7 \\
\hline Gunes et al. & 2006 & turkey & European & $\begin{array}{l}M=67 / 73 \\
F=43 / 77\end{array}$ & $110 / 150$ & $49.22 \pm 1.33 / 48.15 \pm 1.62$ & PCR-RFLP & 7 \\
\hline Moyano et al. & 2007 & Spain & European & $\begin{array}{l}M=22 / 9 \\
F=29 / 12\end{array}$ & $51 / 21$ & $45.5 \pm 13.5 / 48.6 \pm 15.4$ & PCR-RFLP & 5 \\
\hline Wang et al. & 2012 & China & Asian & $\begin{array}{l}M=279 / 263 \\
F=185 / 187\end{array}$ & $464 / 450$ & $50.01 \pm 10.19 / 50.45 \pm 11.22$ & PCR-RFLP & 8 \\
\hline
\end{tabular}


Table 1 Characteristics of studies included in meta-analysis of overall Urolithiasis (Continued)

\begin{tabular}{|c|c|c|c|c|c|c|c|c|}
\hline Study author & Year & Country & Ethnicity & $\begin{array}{l}\text { Sex } \\
\text { cases/controls }\end{array}$ & Total cases/controls & $\begin{array}{l}\text { Age } \\
\text { case/control (Mean) }\end{array}$ & Genotyping method & $\begin{array}{l}\text { Quality } \\
\text { score }\end{array}$ \\
\hline Cakir et al. & 2016 & Turkey & European & $\begin{array}{l}M=65 / 52 \\
F=33 / 18\end{array}$ & $98 / 70$ & $47.2 \pm 16.3 / 42.6 \pm 13.5$ & PCR-RFLP & 6 \\
\hline Goknar et al. & 2016 & Turkey & European & $\begin{array}{l}M=N R \\
F=N R\end{array}$ & $72 / 53$ & $6.94 \pm 3.8 / 7.5 \pm 3.2$ & PCR-RFLP & 6 \\
\hline Subasi et al. & 2017 & turkey & European & $\begin{array}{l}M=26 / 22 \\
F=26 / 29\end{array}$ & $52 / 51$ & $9.8 \pm 3.5 / 10.3 \pm 3.7$ & SNaPshot & 5 \\
\hline Li et al. & 2018 & China & Asian & $\begin{array}{l}M=100 / 60 \\
F=100 / 60\end{array}$ & $200 / 120$ & $35.88 \pm 14.2 / 36.16 \pm 15.20$ & PCR & 7 \\
\hline Yang et al. & 2019 & China & Asian & $\begin{array}{l}M=627 / 614 \\
F=316 / 361\end{array}$ & $943 / 975$ & $51.2 \pm 14.13 / 54.33 \pm 18.11$ & iMLDR & 8 \\
\hline \multicolumn{9}{|l|}{ Apal (rs7975232) } \\
\hline Nishijima et al. & 2002 & Japan & Asian & $\begin{array}{l}M=N R \\
F=N R\end{array}$ & $83 / 83$ & $51.8 \pm 15.6 / 54.4 \pm 13.1$ & PCR-RFLP & 5 \\
\hline Shaogang et al. & 2003 & China & Asian & $\begin{array}{l}M=89 / 58 \\
F=61 / 22\end{array}$ & $150 / 80$ & $43.6 \pm 16 / 49 \pm 19.6$ & PCR-RFLP & 6 \\
\hline Ozkaya et al. & 2003 & Turkey & Asian & $\begin{array}{l}M=26 / 47 \\
F=38 / 43\end{array}$ & $64 / 90$ & $6.7 \pm 3.5 / 7.2 \pm 2.3$ & PCR-RFLP & 5 \\
\hline Rendina et al. & 2004 & Italy & European & $\begin{array}{l}M=94 / 72 \\
F=65 / 52\end{array}$ & $159 / 124$ & $43 \pm 10.8 / 41.9 \pm 10.4$ & PCR-RFLP & 7 \\
\hline Gunes et al. & 2006 & Turkey & European & $\begin{array}{l}M=67 / 73 \\
F=43 / 77\end{array}$ & $110 / 150$ & $49.22 \pm 1.33 / 48.15 \pm 1.62$ & PCR-RFLP & 7 \\
\hline Moyano et al. & 2007 & Spain & European & $\begin{array}{l}M=22 / 9 \\
F=29 / 12\end{array}$ & $51 / 21$ & $45.5 \pm 13.5 / 48.6 \pm 15.4$ & PCR-RFLP & 5 \\
\hline Seo et al. & 2009 & Korea & Asian & $\begin{array}{l}M=88 / 220 \\
F=185 / 305\end{array}$ & $273 / 525$ & 49.9 / 40.1 & PCR-RFLP & 8 \\
\hline Mittal et al. & 2010 & India & Asian & $\begin{array}{l}M=N R \\
F=N R\end{array}$ & $125 / 150$ & $40 \pm 11.5 / 41.5 \pm 10.5$ & PCR-RFLP & 7 \\
\hline Kaysar et al. & 2012 & China & Asian & $\begin{array}{l}M=N R \\
F=N R\end{array}$ & $74 / 103$ & $N R$ & PCR-RFLP & 5 \\
\hline Wang et al. & 2012 & China & Asian & $\begin{array}{l}M=N R \\
F=N R\end{array}$ & $463 / 450$ & $50.01 \pm 10.19 / 50.45 \pm 11.22$ & PCR-RFLP & 8 \\
\hline Cakir et al. & 2016 & Turkey & European & $\begin{array}{l}M=65 / 52 \\
F=33 / 18\end{array}$ & $98 / 70$ & $47.2 \pm 16.3 / 42.6 \pm 13.5$ & PCR-RFLP & 6 \\
\hline Goknar et al. & 2016 & Turkey & European & $\begin{array}{l}M=N R \\
F=N R\end{array}$ & $78 / 60$ & $6.94 \pm 3.8 / 7.5 \pm 3.2$ & PCR-RFLP & 6 \\
\hline Ergon et al. & 2017 & Turkey & European & $\begin{array}{l}M=N R \\
F=N R\end{array}$ & $27 / 13$ & $7.12 \pm 2.64 / 6.92 \pm 2.48$ & Tag-man & 5 \\
\hline Subasi et al. & 2017 & Turkey & European & $\begin{array}{l}M=26 / 22 \\
F=26 / 29\end{array}$ & $52 / 51$ & $9.8 \pm 3.5 / 10.3 \pm 3.7$ & SNaPshot & 5 \\
\hline Li et al. & 2018 & China & Asian & $\begin{array}{l}M=100 / 60 \\
F=100 / 60\end{array}$ & $200 / 120$ & $35.88 \pm 14.2 / 36.16 \pm 15.20$ & PCR & 7 \\
\hline Yang et al. & 2019 & China & Asian & $\begin{array}{l}M=627 / 614 \\
F=316 / 361\end{array}$ & $943 / 975$ & $51.2 \pm 14.13 / 54.33 \pm 18.11$ & iMLDR & 8 \\
\hline
\end{tabular}

Abbreviations: $N R$ not reported, $M$ male, $F$ female

(Fig. 4), indicating that the combined results of our meta-analysis were statistically robust.

\section{Meta-regression analyses}

Potential sources of heterogeneity among included studies was estimated by meta-regression analyses (Table 4). According that, the findings indicated that none of the expected heterogeneity parameter were the source of heterogeneity for the association between VDR gene polymorphism and the risk of urolithiasis (Fig. 5).

\section{Discussion}

In the current most recent meta-analysis, 33 casecontrol association studies evaluating the VDR gene SNPs and urolithiasis risk were analyzed. The results of pooled analysis revealed that none of the four SNPs in 
Table 2 Distribution of genotype and allele among urolithiasis patients and controls

\begin{tabular}{|c|c|c|c|c|c|c|c|c|c|c|c|c|}
\hline \multirow[t]{2}{*}{ Study author } & \multicolumn{5}{|c|}{ Urolithiasis cases } & \multicolumn{5}{|c|}{ Healthy control } & \multirow[t]{2}{*}{ P-HWE } & \multirow[t]{2}{*}{ MAF } \\
\hline & $\mathrm{FF}$ & $\mathrm{Ff}$ & ff & $\mathbf{F}$ & $\mathbf{F}$ & $\mathrm{FF}$ & $\mathrm{Ff}$ & ff & $\mathrm{F}$ & $f$ & & \\
\hline \multicolumn{13}{|l|}{ Fokl (rs2228570) } \\
\hline Chen et al. (b) & 54 & 67 & 25 & 175 & 117 & 21 & 43 & 26 & 85 & 95 & $0 / 43$ & $0 / 527$ \\
\hline Shaogang et al. & 27 & 64 & 59 & 118 & 182 & 17 & 44 & 19 & 78 & 82 & $0 / 36$ & $0 / 512$ \\
\hline Rendina et al. & 69 & 68 & 22 & 206 & 112 & 53 & 55 & 16 & 161 & 87 & $0 / 77$ & $0 / 350$ \\
\hline Mossetti et al. & 43 & 47 & 20 & 133 & 87 & 53 & 55 & 19 & 161 & 93 & $0 / 45$ & $0 / 366$ \\
\hline Relan et al. & 25 & 72 & 53 & 122 & 178 & 38 & 36 & 26 & 112 & 88 & $0 / 01$ & $0 / 44$ \\
\hline Bid et al. (a) & 30 & 106 & 2 & 136 & 90 & 77 & 84 & 5 & 238 & 94 & $0 / 02$ & $0 / 257$ \\
\hline Bid et al. (b) & 11 & 38 & 1 & 60 & 40 & 30 & 28 & 2 & 88 & 32 & $0 / 13$ & $0 / 266$ \\
\hline Liu et al. & 64 & 113 & 58 & 241 & 229 & 58 & 116 & 57 & 232 & 230 & $0 / 94$ & $0 / 497$ \\
\hline Seo et al. & 84 & 134 & 60 & 302 & 254 & 155 & 288 & 92 & 598 & 472 & 0/03 & $0 / 441$ \\
\hline Mittal et al. & 25 & 98 & 2 & 214 & 86 & 69 & 76 & 5 & 148 & 102 & $<0.01$ & $0 / 408$ \\
\hline Basiri et al. & 54 & 42 & 6 & 150 & 54 & 36 & 27 & 43 & 99 & 113 & $<0.01$ & $0 / 533$ \\
\hline Kaysar et al. & 19 & 43 & 12 & 81 & 67 & 33 & 39 & 31 & 105 & 101 & $0 / 01$ & $0 / 490$ \\
\hline Wang et al. & 150 & 234 & 80 & 534 & 394 & 125 & 226 & 99 & 476 & 424 & $0 / 86$ & $0 / 471$ \\
\hline Guha et al. & 78 & 115 & 7 & 271 & 129 & 98 & 90 & 12 & 286 & 114 & $0 / 74$ & $0 / 542$ \\
\hline Cakir et al. & 48 & 38 & 12 & 134 & 62 & 39 & 25 & 6 & 103 & 37 & $0 / 39$ & $0 / 618$ \\
\hline Ergon et al. & 14 & 12 & 1 & 40 & 14 & 7 & 6 & 0 & 20 & 6 & $0 / 27$ & $0 / 230$ \\
\hline Subasi et al. & 23 & 25 & 4 & 71 & 33 & 26 & 21 & 4 & 73 & 29 & $0 / 93$ & $0 / 284$ \\
\hline Li et al. & 38 & 102 & 60 & 178 & 222 & 31 & 72 & 17 & 134 & 106 & $0 / 02$ & $0 / 4416$ \\
\hline Huang et al. & 73 & 49 & 8 & 195 & 65 & 104 & 96 & 24 & 304 & 144 & $0 / 79$ & $0 / 321$ \\
\hline Amar et al. & 136 & 79 & 11 & 351 & 101 & 146 & 77 & 10 & 369 & 97 & 0.37 & 0.519 \\
\hline Study author & \multicolumn{5}{|c|}{ Urolithiasis cases } & \multicolumn{5}{|c|}{ Healthy control } & P-HWE & MAF \\
\hline
\end{tabular}

\section{Taql (rs731236)}

\begin{tabular}{|c|c|c|c|c|c|c|c|c|c|c|c|c|}
\hline Jackman et al. & 6 & 7 & 4 & 19 & 15 & 17 & 8 & 12 & 42 & 32 & 0/82 & $0 / 432$ \\
\hline Nishijima et al. & 49 & 30 & 4 & 128 & 38 & 60 & 22 & 1 & 142 & 24 & $0 / 1$ & $0 / 228$ \\
\hline Ozkaya et al. & 33 & 27 & 4 & 93 & 35 & 50 & 30 & 10 & 130 & 50 & $0 / 81$ & $0 / 277$ \\
\hline Mossetti et al. & 80 & 104 & 36 & 264 & 176 & 35 & 66 & 13 & 136 & 92 & $0 / 53$ & 0/719 \\
\hline Shaogang et al. & 52 & 74 & 24 & 178 & 122 & 33 & 36 & 11 & 102 & 58 & $0 / 3$ & $0 / 362$ \\
\hline Mossetti et al. & 21 & 53 & 36 & 95 & 125 & 21 & 68 & 38 & 110 & 144 & $0 / 39$ & $0 / 566$ \\
\hline Gunes et al. & 37 & 63 & 10 & 137 & 83 & 61 & 73 & 16 & 195 & 105 & $0 / 02$ & $0 / 35$ \\
\hline Shayan et al. & 27 & 35 & 18 & 89 & 71 & 13 & 25 & 2 & 51 & 29 & $0 / 74$ & $0 / 362$ \\
\hline Moyano et al. & 15 & 23 & 13 & 53 & 49 & 9 & 10 & 2 & 28 & 14 & $<0.01$ & $0 / 333$ \\
\hline Seo et al. & 252 & 23 & 3 & 527 & 29 & 487 & 39 & 7 & 1013 & 53 & 0/05 & 0/049 \\
\hline Mittal et al. & 56 & 61 & 8 & 173 & 77 & 84 & 50 & 16 & 218 & 82 & 0/03 & $0 / 273$ \\
\hline Basiri et al. & 41 & 50 & 11 & 132 & 72 & 52 & 37 & 17 & 141 & 71 & $0 / 77$ & $0 / 334$ \\
\hline Wang et al. & 430 & 32 & 2 & 892 & 36 & 414 & 35 & 1 & 863 & 37 & 0/08 & 0/041 \\
\hline Aykan et al. & 67 & 61 & 36 & 195 & 133 & 66 & 86 & 15 & 218 & 116 & $<0.01$ & $0 / 347$ \\
\hline Guha et al. & 58 & 82 & 60 & 196 & 202 & 65 & 58 & 77 & 188 & 212 & $0 / 67$ & $0 / 349$ \\
\hline Rendina et al. & 186 & 158 & 28 & 530 & 214 & 31 & 44 & 13 & 106 & 70 & $0 / 16$ & $0 / 473$ \\
\hline Cakir et al. & 35 & 44 & 19 & 114 & 82 & 31 & 29 & 10 & 91 & 49 & $0 / 43$ & 0/173 \\
\hline Goknar et al. & 25 & 41 & 12 & 91 & 65 & 14 & 43 & 3 & 71 & 49 & 0/83 & $0 / 408$ \\
\hline Subasi et al. & 4 & 25 & 23 & 33 & 71 & 9 & 24 & 18 & 42 & 60 & 0/77 & $0 / 588$ \\
\hline
\end{tabular}


Table 2 Distribution of genotype and allele among urolithiasis patients and controls (Continued)

\begin{tabular}{|c|c|c|c|c|c|c|c|c|c|c|c|c|}
\hline Li et al. & 189 & 11 & 0 & 389 & 11 & 114 & 6 & 0 & 234 & 6 & $0 / 82$ & $0 / 025$ \\
\hline Yang et al. & 849 & 92 & 2 & 1790 & 96 & 870 & 103 & 2 & 1843 & 107 & $0 / 67$ & $0 / 471$ \\
\hline Amar et al. & 112 & 86 & 29 & 310 & 144 & 116 & 104 & 23 & 336 & 150 & $0 / 42$ & $0 / 149$ \\
\hline \multirow[t]{2}{*}{ Study author } & \multicolumn{5}{|c|}{ Urolithiasis cases } & \multicolumn{5}{|c|}{ Healthy control } & P-HWE & MAF \\
\hline & BB & $\mathrm{Bb}$ & bb & B & b & BB & $\mathrm{Bb}$ & bb & B & b & & \\
\hline \multicolumn{13}{|l|}{ Bsml (rs1544410) } \\
\hline Ruggiero et al. & 4 & 12 & 11 & 19 & 35 & 18 & 108 & 24 & 144 & 156 & $<0.01$ & $0 / 52$ \\
\hline Chen et al. (a) & 110 & 10 & 4 & 230 & 18 & 78 & 9 & 3 & 165 & 15 & $<0.01$ & 0/083 \\
\hline Ozkaya et al. & 5 & 36 & 23 & 46 & 82 & 13 & 49 & 28 & 75 & 105 & $0 / 25$ & $0 / 583$ \\
\hline Rendina et al. & 47 & 69 & 43 & 163 & 155 & 39 & 56 & 29 & 134 & 114 & $0 / 31$ & $0 / 459$ \\
\hline Mossetti et al. & 40 & 46 & 24 & 126 & 94 & 40 & 56 & 31 & 136 & 118 & $0 / 2$ & $0 / 464$ \\
\hline Relan et al. & 48 & 62 & 40 & 158 & 142 & 46 & 28 & 26 & 120 & 80 & $<0.01$ & $0 / 40$ \\
\hline Gunes et al. & 15 & 64 & 31 & 94 & 126 & 19 & 75 & 56 & 113 & 187 & $0 / 42$ & $0 / 623$ \\
\hline Moyano et al. & 5 & 25 & 21 & 35 & 67 & 5 & 9 & 7 & 19 & 23 & $0 / 53$ & $0 / 547$ \\
\hline Wang et al. & 3 & 66 & 395 & 72 & 856 & 2 & 70 & 378 & 74 & 826 & $0 / 51$ & 0/917 \\
\hline Cakir et al. & 43 & 40 & 15 & 126 & 70 & 26 & 34 & 10 & 86 & 54 & $0 / 57$ & $0 / 476$ \\
\hline Goknar et al. & 21 & 35 & 16 & 77 & 67 & 16 & 37 & 0 & 69 & 37 & 0/01 & $0 / 349$ \\
\hline Subasi et al. & 28 & 19 & 5 & 75 & 29 & 20 & 23 & 8 & 63 & 39 & $0 / 74$ & $0 / 382$ \\
\hline Li et al. & 181 & 19 & 0 & 381 & 19 & 111 & 9 & 0 & 231 & 9 & $0 / 67$ & 0/0375 \\
\hline Yang et al. & 65 & 394 & 484 & 524 & 1362 & 78 & 417 & 480 & 573 & 1377 & $0 / 28$ & $0 / 315$ \\
\hline \multirow[t]{2}{*}{ Study author } & \multicolumn{5}{|c|}{ Urolithiasis cases } & \multicolumn{5}{|c|}{ Healthy control } & P-HWE & MAF \\
\hline & AA & $\mathrm{Aa}$ & aa & A & a & AA & $\mathrm{Aa}$ & aa & A & a & & \\
\hline \multicolumn{13}{|l|}{ Apal (rs7975232) } \\
\hline Nishijima et al. & 14 & 34 & 35 & 62 & 104 & 9 & 37 & 37 & 55 & 111 & $0 / 25$ & $0 / 626$ \\
\hline Shaogang et al. & 32 & 69 & 49 & 133 & 167 & 11 & 38 & 31 & 60 & 100 & $0 / 9$ & $0 / 625$ \\
\hline Ozkaya et al. & 13 & 30 & 21 & 56 & 72 & 4 & 50 & 36 & 58 & 122 & 0/09 & $0 / 677$ \\
\hline Rendina et al. & 43 & 87 & 29 & 173 & 145 & 37 & 68 & 19 & 142 & 106 & $0 / 18$ & $0 / 427$ \\
\hline Gunes et al. & 40 & 58 & 12 & 138 & 82 & 59 & 72 & 19 & 190 & 110 & $0 / 68$ & $0 / 366$ \\
\hline Moyano et al. & 11 & 29 & 11 & 51 & 51 & 7 & 9 & 5 & 23 & 19 & $0 / 53$ & $0 / 452$ \\
\hline Seo et al. & 152 & 84 & 37 & 388 & 158 & 282 & 192 & 51 & 756 & 294 & $0 / 03$ & $0 / 28$ \\
\hline Mittal et al. & 43 & 70 & 12 & 156 & 94 & 57 & 71 & 22 & 185 & 115 & $0 / 98$ & 0/383 \\
\hline Kaysar et al.. & 21 & 29 & 24 & 71 & 77 & 32 & 42 & 29 & 106 & 100 & 0/06 & $0 / 485$ \\
\hline Wang et al & 27 & 177 & 259 & 231 & 695 & 46 & 195 & 209 & 287 & 613 & $0 / 75$ & $0 / 748$ \\
\hline Cakir et al. & 43 & 40 & 15 & 126 & 70 & 26 & 34 & 10 & 86 & 54 & $0 / 63$ & $0 / 135$ \\
\hline Goknar et al. & 24 & 42 & 12 & 90 & 66 & 11 & 40 & 9 & 62 & 58 & 0/01 & $0 / 483$ \\
\hline Ergon et al. & 9 & 12 & 6 & 30 & 24 & 4 & 6 & 3 & 14 & 12 & 0/79 & $0 / 461$ \\
\hline Subasi et al. & 18 & 24 & 10 & 60 & 44 & 22 & 14 & 15 & 58 & 44 & 0/01 & $0 / 431$ \\
\hline Li et al. & 73 & 87 & 40 & 233 & 167 & 57 & 51 & 12 & 165 & 75 & $0 / 9$ & $0 / 312$ \\
\hline Yang et al. & 65 & 394 & 484 & 524 & 1362 & 78 & 417 & 480 & 573 & 1377 & $0 / 49$ & $0 / 743$ \\
\hline
\end{tabular}

Abbreviations: P-HWE $p$-value for Hardy-Weinberg equilibrium, MAF minor allele frequency of control group

$V D R$ gene were in significant association with proneness to urolithiasis. That notwithstanding, subgroup analysis based on the population stratification demonstrated increased risk of urolithiasis in East-Asian (recessive, allelic and aa vs. AA model) and Caucasian (heterozygous model) population with ApaI and TaqI gene polymorphism, respectively.

Several investigations have noted that $V D R$ gene SNPs have been contributing genetic factors in susceptibility to urolithiasis [27, 31, 34, 49]. A bulk of studies have 


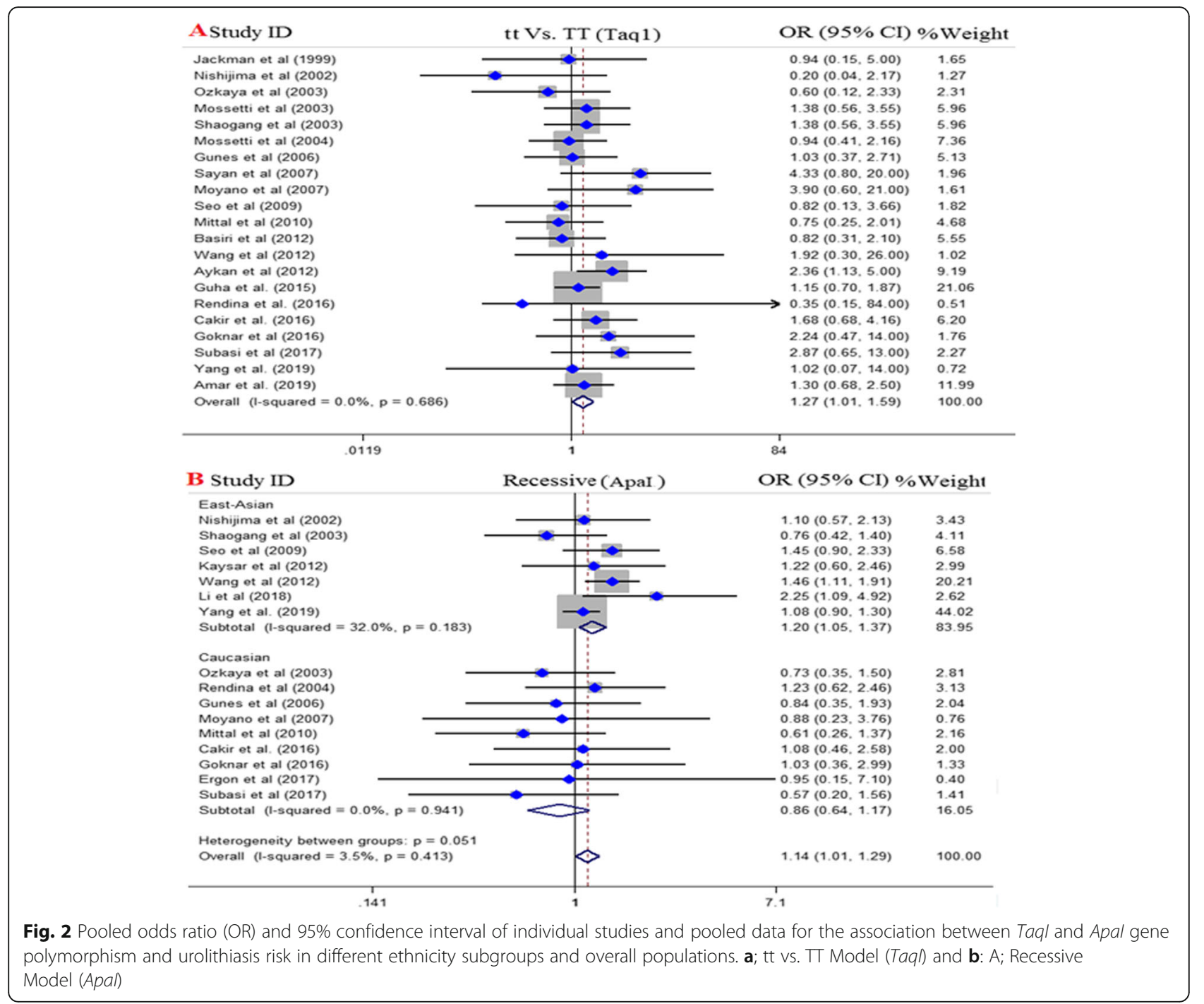

attempted to disclose the possible association between $V D R$ gene SNPs and urolithiasis risk; that notwithstanding, the findings still show discrepancies and a comprehensive meta-analysis seems to be required to shed insights on the unknown conundrums. As a result, we performed a meta-analysis to investigate the consequence of the common four SNPs in the VDR gene, namely FokI (rs2228570), TaqI (rs731236), BsmI (rs1544410), and ApaI (rs7975232) on the risk of urolithiasis. The discrepancies in outcome among the various ethnicities might be due to differences in geographic and ethnical diversity, and impression of ethnicity on the serum level of vitamin $\mathrm{D}$ as well as the $V D R$ gene expression [69].

Reports have shown the role of environmental factors on the risk of different diseases. For example, seasonal differences may impress the serum level of vitamin D [70]. Among the pregnant women in south-eastern USA, season was indicated to be associated with vitamin D levels in non-Hispanic women [71]. Sun exposure has been shown to interact with functional variants of the $V D R$ gene [72]. Additionally, sun exposure and the differences between high and low latitudes, it has been implied that people in high latitude regions experience lower levels of vitamin D, especially in those with darker skin (which is a natural barrier to the UV radiation) [73]. As a result, environmental stimuli may impress the functional variants of the $V D R$ gene as well as serum levels of vitamin $\mathrm{D}$ and, hence, modify the risk of urolithiasis susceptibility, along with VDR genetic polymorphisms.

Vitamin D is a critical hormone and play a role in the metabolism of calcium. This vitamin implements its function by binding to the VDR. The genetic variations in the $V D R$ gene have been shown to impress the interactions of the vitamin D/VDR, modulating the susceptibility risk for several pathologic conditions. FokI polymorphism can modulate the ATG start cordon in 
Table 3 Main results of pooled ORs in meta-analysis of VDR gene polymorphisms

\begin{tabular}{|c|c|c|c|c|c|c|c|c|c|c|}
\hline \multirow[t]{2}{*}{ Subgroup } & \multirow[b]{2}{*}{ Genetic model } & \multirow{2}{*}{$\begin{array}{l}\text { Sample size } \\
\text { Case/Control }\end{array}$} & \multicolumn{2}{|c|}{ Test of association } & \multicolumn{2}{|c|}{$\begin{array}{l}\text { Test of } \\
\text { heterogeneity }\end{array}$} & \multicolumn{2}{|c|}{$\begin{array}{l}\text { Test of publication bias } \\
\text { (Begg's test) }\end{array}$} & \multicolumn{2}{|c|}{$\begin{array}{l}\text { Test of publication bias } \\
\text { (Egger's test) }\end{array}$} \\
\hline & & & $\overline{\mathrm{OR}}$ & 95\%Cl (p-value) & $\overline{1^{2}(\%)}$ & $P$ & $\bar{Z}$ & $P$ & $\bar{T}$ & $P$ \\
\hline \multicolumn{11}{|c|}{ Fokl (rs2228570) } \\
\hline \multirow[t]{5}{*}{ Overall } & Dominant model & $3114 / 3174$ & 1.16 & $0.90-1.50(0.25)$ & 77.7 & $\leq 0.001$ & 1.73 & 0.08 & 1.37 & 0.19 \\
\hline & Recessive model & $3114 / 3174$ & 0.92 & $0.68-1.25(0.58)$ & 67.2 & $\leq 0.001$ & -1.17 & 0.24 & -0.68 & 0.50 \\
\hline & Allelic model & $3114 / 3174$ & 1.02 & $0.86-1.22(0.82)$ & 78.5 & $\leq 0.001$ & 0.25 & 0.80 & 0.45 & 0.66 \\
\hline & ff vs. FF & $3114 / 3174$ & 1.10 & $0.72-1.69(0.65)$ & 77.9 & $\leq 0.001$ & -0.72 & 0.47 & -0.14 & 0.88 \\
\hline & Ff vs. FF & $3114 / 3174$ & 1.12 & $0.88-1.43(0.34)$ & 74.1 & $\leq 0.001$ & 1.94 & 0.05 & 1.39 & 0.18 \\
\hline \multirow[t]{5}{*}{ East-Asian } & Dominant model & $1677 / 1833$ & 0.91 & $0.77-1.06(0.22)$ & 1 & 0.42 & 0.99 & 0.32 & 1.64 & 0.15 \\
\hline & Recessive model & $1677 / 1833$ & 0.98 & $0.66-1.45(0.91)$ & 74.9 & $\leq 0.001$ & 0 & 1 & -0.18 & 0.86 \\
\hline & Allelic model & $1677 / 1833$ & 0.95 & $0.78-1.16(0.62)$ & 71.6 & $\leq 0.001$ & -0.25 & 0.80 & 0.15 & 0.88 \\
\hline & ff vs. FF & $1677 / 1833$ & 0.93 & $0.61-1.40(0.71)$ & 67.7 & $\leq 0.001$ & 0 & 1 & 0.10 & 0.92 \\
\hline & Ff vs. FF & 1677 / 1833 & 0.88 & $0.74-1.04(0.12)$ & 0 & 0.48 & 0.78 & 0.45 & 0.84 & 0.43 \\
\hline \multirow[t]{5}{*}{ Caucasian } & Dominant model & $1437 / 1341$ & 1.33 & $0.87-2.05(0.18)$ & 83.6 & $\leq 0.001$ & 1.04 & 0.29 & 0.62 & 0.55 \\
\hline & Recessive model & $1437 / 1341$ & 0.84 & $0.49-1.44(0.52)$ & 62.8 & $\leq 0.001$ & -1.73 & 0.08 & -0.69 & 0.51 \\
\hline & Allelic model & 1437 / 1341 & 1.08 & $0.81-1.45(0.59)$ & 82.1 & $\leq 0.001$ & -0.21 & 0.83 & 0.2 & 0.82 \\
\hline & ff vs. FF & $1437 / 1341$ & 1.28 & $0.56-2.94(0.55)$ & 81 & $\leq 0.001$ & -0.25 & 0.80 & -0.68 & 0.52 \\
\hline & Ff vs. FF & 1437 / 1341 & 1.33 & $0.90-1.98(0.15)$ & 75.5 & $\leq 0.001$ & 1.25 & 0.21 & 0.56 & 0.59 \\
\hline \multicolumn{11}{|c|}{ Taql (rs731236) } \\
\hline \multirow[t]{5}{*}{ Overall } & Dominant model & 4188 / 3955 & 1.05 & $0.93-1.19(0.41)$ & 14 & 0.27 & 0.45 & 0.65 & 0.40 & 0.69 \\
\hline & Recessive model & 4188 / 3955 & 1.07 & $0.88-1.30(0.48)$ & 31.5 & 0.08 & 0.05 & 0.96 & -0.23 & 0.83 \\
\hline & Allelic model & 4188 / 3955 & 1.06 & $0.97-1.16(0.23)$ & 2.6 & 0.42 & -0.18 & 0.85 & -0.21 & 0.83 \\
\hline & tt vs. Tा & 4188 / 3955 & 1.27 & $1.01-1.59(0.04)$ & 0 & 0.68 & 0.05 & 0.96 & -0.06 & 0.95 \\
\hline & Tt vs. TT & 4188 / 3955 & 1.04 & $0.91-1.18(0.59)$ & 34.5 & 0.05 & 0.45 & 0.65 & 0.33 & 0.74 \\
\hline \multirow[t]{5}{*}{ East-Asian } & Dominant model & $2118 / 2241$ & 0.94 & $0.76-1.16(0.55)$ & 0 & 0.56 & 0.19 & 0.85 & -0.19 & 0.85 \\
\hline & Recessive model & $2118 / 2241$ & 0.97 & $0.50-1.88(0.92)$ & 0 & 0.68 & -0.49 & 0.62 & -1.45 & 0.24 \\
\hline & Allelic model & $2118 / 2241$ & 0.95 & $0.79-1.15(0.60)$ & 0 & 0.51 & -1.69 & 0.09 & -1.27 & 0.27 \\
\hline & tt vs. Tा & $2118 / 2241$ & 1.02 & $0.51-2.02(0.96)$ & 0 & 0.50 & -0.98 & 0.32 & -1.70 & 0.18 \\
\hline & Tt vs. TT & $2118 / 2241$ & 0.94 & $0.76-1.16(0.26)$ & 0 & 0.67 & 0.56 & 0.57 & 0.37 & 0.73 \\
\hline \multirow[t]{5}{*}{ Caucasian } & Dominant model & $2070 / 1714$ & 1.12 & $0.96-1.29(0.15)$ & 20.3 & 0.22 & 0.09 & 0.92 & -0.03 & 0.97 \\
\hline & Recessive model & $2070 / 1714$ & 1.08 & $0.88-1.33(0.44)$ & 44.1 & 0.03 & 1.16 & 0.24 & 0.92 & 0.38 \\
\hline & Allelic model & $2070 / 1714$ & 1.09 & $0.98-1.21(0.09)$ & 4.5 & 0.40 & 1.34 & 0.18 & 1.58 & 0.15 \\
\hline & tt vs. Tा & $2070 / 1714$ & 1.30 & $1.02-1.65(0.03)$ & 0 & 0.62 & 1.52 & 0.12 & 2.35 & 0.04 \\
\hline & Tt vs. TT & $2070 / 1714$ & 1.10 & $0.93-1.29(0.56)$ & 45.7 & 0.02 & -0.80 & 0.42 & -0.41 & 0.69 \\
\hline \multicolumn{11}{|c|}{ Bsml (rs1544410) } \\
\hline \multirow[t]{5}{*}{ Overall } & Dominant model & $3065 / 2915$ & 0.97 & $0.84-1.12(0.69)$ & 12 & 0.31 & 0.41 & 0.68 & -0.04 & 0.96 \\
\hline & Recessive model & $3065 / 2915$ & 0.98 & $0.86-1.12(0.74)$ & 38.7 & 0.06 & 0.27 & 0.78 & 0.46 & 0.65 \\
\hline & Allelic model & $3065 / 2915$ & 0.99 & $0.91-1.08(0.82)$ & 42.5 & 0.03 & 0.55 & 0.58 & 0.74 & 0.47 \\
\hline & bb vs. BB & $3065 / 2915$ & 0.95 & $0.79-1.14(0.56)$ & 22.2 & 0.21 & 0.27 & 0.78 & 0.10 & 0.92 \\
\hline & $\mathrm{Bb}$ vs. BB & $3065 / 2915$ & 0.97 & $0.83-1.14(0.74)$ & 0.8 & 0.44 & 0 & 1 & 0.38 & 0.71 \\
\hline \multirow[t]{4}{*}{ East-Asian } & Dominant model & $1783 / 1686$ & 0.86 & $0.71-1.05(0.41)$ & 0 & 0.76 & 0.52 & 0.60 & -0.47 & 0.72 \\
\hline & Recessive model & $1783 / 1686$ & 0.88 & $0.73-1.05(0.16)$ & 0 & 0.59 & -1.00 & 0.31 & - & - \\
\hline & Allelic model & $1783 / 1686$ & 0.89 & $0.79-1.01(0.06)$ & 0 & 0.58 & 0.52 & 0.60 & -0.04 & 0.97 \\
\hline & bb vs. BB & 1783 / 1686 & 0.78 & $0.60-1.00(0.05)$ & 0 & 0.88 & -1.00 & 0.31 & - & - \\
\hline
\end{tabular}


Table 3 Main results of pooled ORs in meta-analysis of VDR gene polymorphisms (Continued)

\begin{tabular}{|c|c|c|c|c|c|c|c|c|c|c|}
\hline \multirow[t]{2}{*}{ Subgroup } & \multirow[b]{2}{*}{ Genetic model } & \multirow{2}{*}{$\begin{array}{l}\text { Sample size } \\
\text { Case/Control }\end{array}$} & \multicolumn{2}{|c|}{ Test of association } & \multicolumn{2}{|c|}{$\begin{array}{l}\text { Test of } \\
\text { heterogeneity }\end{array}$} & \multicolumn{2}{|c|}{$\begin{array}{l}\text { Test of publication bias } \\
\text { (Begg's test) }\end{array}$} & \multicolumn{2}{|c|}{$\begin{array}{l}\text { Test of publication bias } \\
\text { (Egger's test) }\end{array}$} \\
\hline & & & $\overline{\mathrm{OR}}$ & 95\%Cl ( $\boldsymbol{p}$-value) & $\overline{1^{2}(\%)}$ & $P$ & $\bar{Z}$ & $P$ & $\bar{T}$ & $P$ \\
\hline & $\mathrm{Bb}$ vs. BB & $1783 / 1686$ & 0.91 & $0.73-1.12(0.36)$ & 0 & 0.81 & -0.52 & 0.60 & 0.87 & 0.54 \\
\hline \multirow[t]{5}{*}{ Caucasian } & Dominant model & $1282 / 1229$ & 1.11 & $0.90-1.36(0.34)$ & 18.6 & 0.26 & 0 & 1 & 0.22 & 0.83 \\
\hline & Recessive model & $1282 / 1229$ & 1.11 & $0.91-1.35(0.30)$ & 45.3 & 0.05 & 0.49 & 0.62 & 0.81 & 0.45 \\
\hline & Allelic model & $1282 / 1229$ & 1.10 & $0.97-1.24(0.12)$ & 43.2 & 0.06 & 0.83 & 0.40 & 0.92 & 0.38 \\
\hline & bb vs. BB & $1282 / 1229$ & 1.16 & $0.89-1.50(0.26)$ & 21.5 & 0.24 & 0.99 & 0.32 & 0.47 & 0.65 \\
\hline & $\mathrm{Bb}$ vs. BB & $1282 / 1229$ & 1.06 & $0.84-1.32(0.63)$ & 20.7 & 0.24 & -0.21 & 0.83 & -0.09 & 0.93 \\
\hline \multicolumn{11}{|c|}{ Apal (rs7975232) } \\
\hline \multirow[t]{5}{*}{ Overall } & Dominant model & $2950 / 3065$ & 1.08 & $0.93-1.25(0.30)$ & 48.6 & 0.01 & -0.35 & 0.72 & -0.54 & 0.60 \\
\hline & Recessive model & $2950 / 3065$ & 1.14 & $1.01-1.29(0.03)$ & 3.5 & 0.41 & -0.38 & 0.70 & -0.02 & 0.98 \\
\hline & Allelic model & $2950 / 3065$ & 1.09 & $1.00-1.18(0.05)$ & 31 & 0.11 & -0.64 & 0.52 & 0.42 & 0.67 \\
\hline & aa vs. AA & $2950 / 3065$ & 1.21 & $1.00-1.47(0.05)$ & 27.5 & 0.14 & -1.15 & 0.25 & -0.85 & 0.41 \\
\hline & Aa vs. AA & $2950 / 3065$ & 1.10 & $0.94-1.28(0.29)$ & 41.1 & 0.04 & -0.94 & 0.34 & -0.39 & 0.70 \\
\hline \multirow[t]{5}{*}{ East-Asian } & Dominant model & $2186 / 2336$ & 1.15 & $0.96-1.38(0.12)$ & 38.8 & 0.13 & 0.19 & 0.85 & 0.21 & 0.84 \\
\hline & Recessive model & $2186 / 2336$ & 1.20 & $1.05-1.37(\leq 0.001)$ & 32 & 0.18 & 1.69 & 0.09 & 1.37 & 0.24 \\
\hline & Allelic model & $2186 / 2336$ & 1.15 & $1.05-1.26(\leq 0.001)$ & 49.1 & 0.06 & 0.19 & 0.85 & -0.09 & 0.93 \\
\hline & aa vs. AA & $2186 / 2336$ & 1.40 & $1.12-1.75(\leq 0.001)$ & 36.1 & 0.15 & -0.19 & 0.85 & 0.45 & 0.67 \\
\hline & Aa vs. AA & $2186 / 2336$ & 1.10 & $0.90-1.33(0.35)$ & 40.7 & 0.12 & 0.56 & 0.57 & 0.54 & 0.62 \\
\hline \multirow[t]{5}{*}{ Caucasian } & Dominant model & 764 / 729 & 0.96 & $0.75-1.22(0.73)$ & 55.4 & 0.02 & -0.83 & 0.40 & -1.14 & 0.29 \\
\hline & Recessive model & 764 / 729 & 0.86 & $0.64-1.17(0.34)$ & 0 & 0.94 & 0.49 & 0.62 & -0.25 & 0.81 \\
\hline & Allelic model & 764 / 729 & 0.94 & $0.80-1.09(0.40)$ & 0 & 0.75 & 0.42 & 0.67 & 0.60 & 0.56 \\
\hline & aa vs. AA & 764 / 729 & 0.83 & $0.58-1.20(0.32)$ & 0 & 0.68 & -0.99 & 0.32 & -1.32 & 0.23 \\
\hline & Aa vs. AA & 764 / 729 & 1.10 & $0.85-1.42(0.45)$ & 47.9 & 0.05 & -1.25 & 0.21 & -1.40 & 0.20 \\
\hline
\end{tabular}

the VDR protein and BsmI SNP can modify the VDR protein expression $[74,75]$. Additionally, ApaI and TaqI SNPs have been shown to have potential to modify the mRNA transcription of $V D R$ gene and can modulate the stability of VDR mRNA [21]. FokI SNP has been shown to have potential to modulate the function of transcription factors $[76,77]$.

A recent meta-analysis by González-Castro in 2019 [78], including 23 studies (a total of 1536 cases/1767 controls for ApaI polymorphism, 1571 cases/ 1455 controls for BsmI polymorphism, 2145 cases/2280 controls for FokI polymorphism, and 2160 cases/2307 controls for TaqI polymorphism), indicated that BsmI polymorphism had a protective association with nephrolithiasis in the allelic and homozygous models. Moreover, both TaqI polymorphism and FokI polymorphism were associated with a decreased risk of nephrolithiasis in the heterozygous model. However, no association of ApaI polymorphism was detected with nephrolithiasis. However, our most recent update metaanalysis in 2020, by including 33 studies (a total of 2950 cases/3065 controls for ApaI polymorphism, 3065 cases/ 2915 controls for BsmI polymorphism, 3114 cases/3174 controls for FokI polymorphism, and 4188 cases/3955 controls for TaqI polymorphism), indicated that none of the VDR gene polymorphisms mentioned above were associated significantly with nephrolithiasis risk in the overall analysis except ApaI SNP. However, our subgroup analysis according to population stratification revealed that ApaI gene polymorphism increased risk of urolithiasis in East-Asian patients by the recessive, allelic and homozygous model and TaqI gene SNP in Caucasians population through the heterozygous model. On the other hand, a meta-analysis in 2014 with respect to the study of the associations between VDR gene SNPs and urolithiasis risk included 20 studies in the analysis [53]. They found that the TaqI polymorphism was associated with an increased risk of urolithiasis, whereas the ApaI, BsmI, and FokI polymorphisms did not show any significant association. Moreover, stratifying for ethnicity, a slightly increased risk was found among Asians as compared with Whites for TaqI SNP. On the other hand, our meta-analysis on 33 studies did not result in any strong significant association between all four SNPs and urolithiasis risk in the pooled overall comparison. However, subgroup analysis demonstrated a significant 

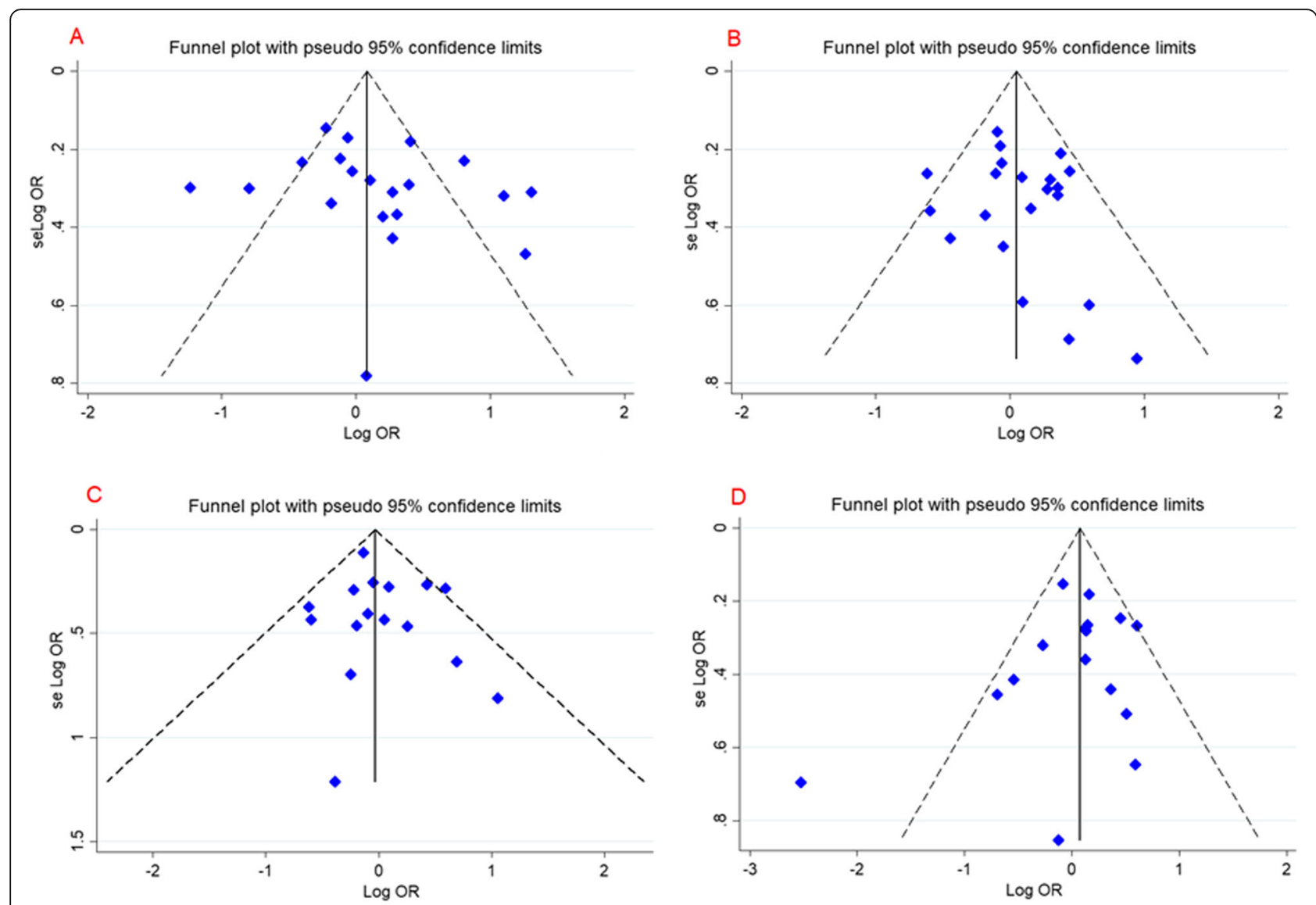

Fig. 3 Begg's funnel plot for publication bias test. a; Dominant Model Fokl, b; Dominant Model Taql, c; Dominant Model Bsml, d; Dominant Model Apal. Each point represents a separate study for the indicated association

increased risk of urolithiasis in East-Asian and Caucasians populations in association with ApaI and TaqI genes polymorphism. In the current meta-analysis, thirteen more studies were added in comparison to the previous study, and did not support the previous finding in the overall analysis.

The subgroup analyses were conducted based on the ethnicity to identify the potential impression of the genetic background on the association of VDR gene polymorphisms and urolithiasis. Our analysis resulted in identification of ApaI and TaqI polymorphism association with increased risk of urolithiasis in East-Asian and Caucasians populations. However, the previous meta-analysis identified the same association in only Asians [53]. These discrepancies may stem from diversities in the genetic backgrounds. Furthermore, given that solar UV radiation is involved in the process of vitamin D generation [79], the significant association of $V D R$ gene TaqI SNP in Asians might be attributed to the partially higher amount of exposure to UVR [80]. Moreover, it has been implied that level of UV exposure may impress that the associations between $V D R$ gene polymorphisms and disorders. In patients with nonHodgkin lymphoma, it was reported that patients with CC genotype for TaqI SNP who experienced sun exposure less than $7 \mathrm{~h}$ per week exhibited higher risk of the disease in comparison to patients with TT genotype with the similar duration of sun exposure [81]. In addition, reports showed that the TaqI $\mathrm{T}$ allele was more common in prostate cancer patients in a southern European population compared with the controls [82]. Plus, in a British population, the association of FokI polymorphism was observed to be limited to cases with a high exposed to UV [83]. Other than that, gender has been known as also a major risk factor for urolithiasis risk. It was shown that the FokI polymorphism had significant differences in females but not males, implying to the role of gender on the function of VDR [44]. Nonetheless, lack of sufficient data hindered the subgroup analysis based on gender in the current meta-analysis, which need to be addressed in the further studies.

Data from GWASs as well as association studies in different ethnic groups have revealed that VDR gene polymorphisms play a role in altering the risk of urolithiasis 


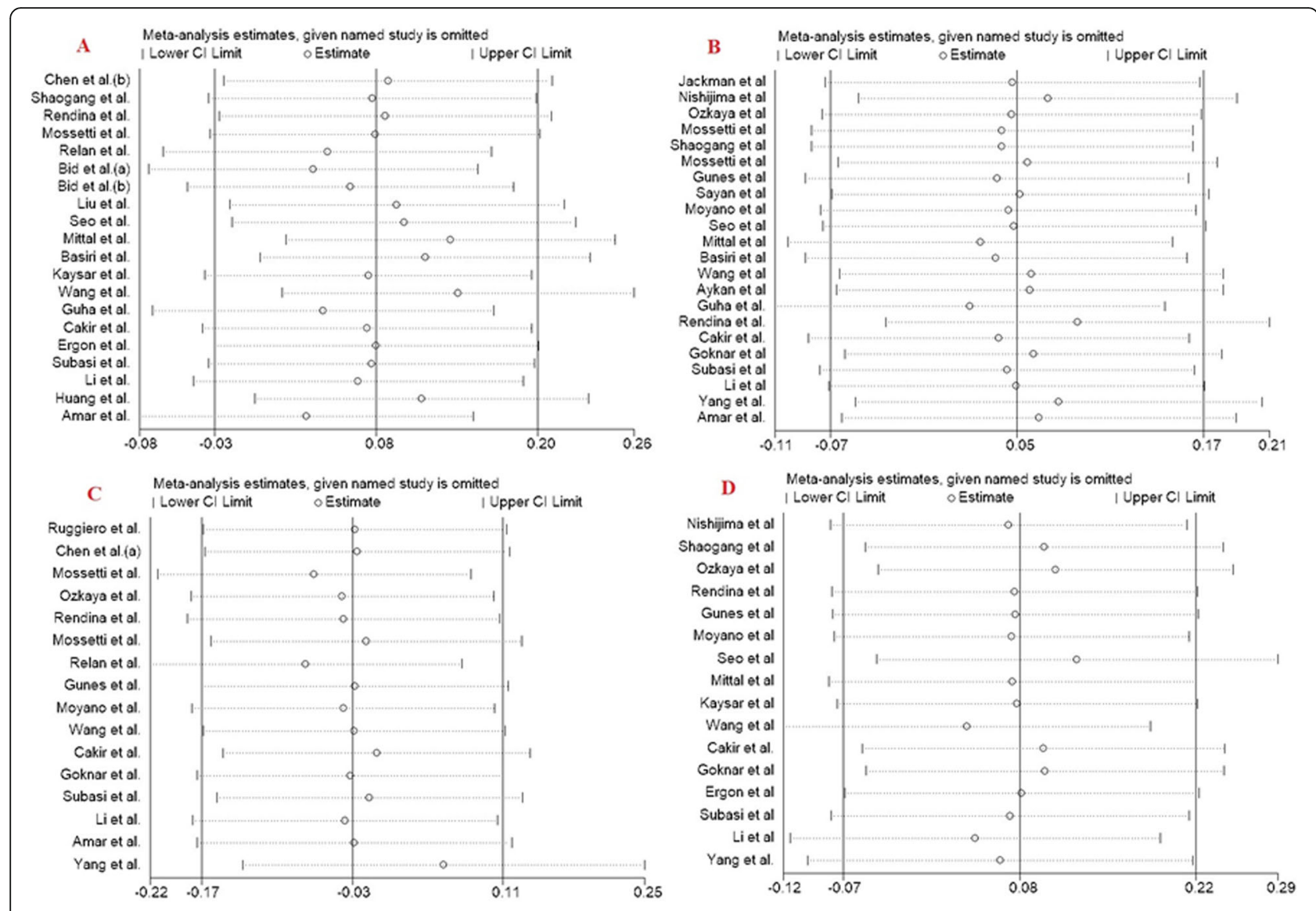

Fig. 4 Sensitivity analysis in present meta-analysis investigates the single nucleotide polymorphisms of Vitamin $D$ Receptor contribute to risk for urolithiasis susceptibility (a, Fokl; b, Taql; c, Bsml; d, Apal)

Table 4 Meta-regression analyses of potential source of heterogeneity

\begin{tabular}{|c|c|c|c|c|c|c|c|}
\hline \multicolumn{2}{|c|}{ Heterogeneity Factor } & \multirow[t]{2}{*}{ Coefficient } & \multirow[t]{2}{*}{ SE } & \multirow[t]{2}{*}{ T-test } & \multirow[t]{2}{*}{$\boldsymbol{P}$-value } & \multicolumn{2}{|l|}{$95 \% \mathrm{Cl}$} \\
\hline & & & & & & UL & $\mathrm{LL}$ \\
\hline \multicolumn{8}{|l|}{ Fokl } \\
\hline \multirow[t]{2}{*}{ Dominant } & Publication Year & -0.031 & 0.03 & -0.87 & 0.39 & -0.108 & 0.045 \\
\hline & Genotyping Method & -0.032 & 0.16 & -0.20 & 0.84 & -0.370 & 0.306 \\
\hline \multicolumn{8}{|l|}{ Taql } \\
\hline \multirow[t]{2}{*}{ Dominant } & Publication Year & -0.011 & 0.01 & -0.86 & 0.40 & -0.037 & 0.015 \\
\hline & Genotyping Method & 0.018 & 0.04 & 0.42 & 0.67 & -0.073 & 0.109 \\
\hline \multicolumn{8}{|l|}{ Bsml } \\
\hline \multirow[t]{2}{*}{ Dominant } & Publication Year & -0.025 & 0.013 & -1.93 & 0.07 & -0.054 & 0.002 \\
\hline & Genotyping Method & -0.056 & 0.58 & -0.97 & 0.34 & -0.181 & 0.068 \\
\hline \multicolumn{8}{|l|}{ Apal } \\
\hline \multirow[t]{2}{*}{ Dominant } & Publication Year & 0.012 & 0.018 & 0.68 & 0.50 & -0.026 & 0.051 \\
\hline & Genotyping Method & 0.050 & 0.051 & 0.99 & 0.34 & -0.059 & 0.160 \\
\hline
\end{tabular}




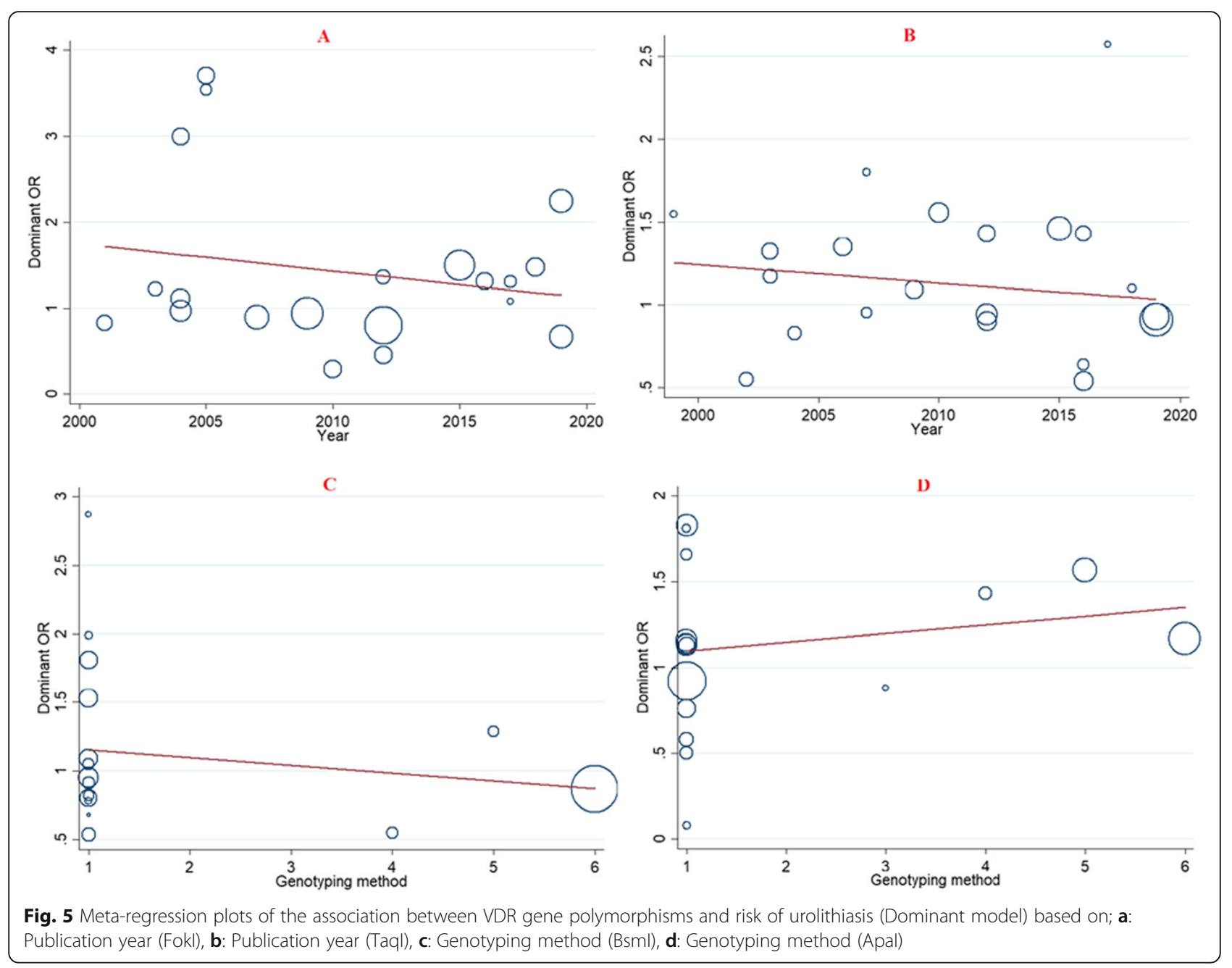

development. Although our analysis did not endorse the association of VDR gene BsmI, ApaI, FokI, and TaqI SNPs with susceptibility to urolithiasis, the gene can be of beneficial applications in populations with significant associations. Generally, the concept of personalized medicine has been widely accepted, implying to the consideration of genetic makeup of each patient in approaching with optimized medication. As a consequence, clarification of VDR gene polymorphisms contribution to the urolithiasis predisposition could be advantageous in clinics with respect to better diagnosis of subjects at risk as well as treatment with maximum efficacy.

Despite we tried to perform the possibly well-suited analysis of the available data, a number of caveats and confining factors are related to this meta-analysis. First, our literature search was limited to only English-written papers, raising the chance of excluding of potentially worthwhile findings. Second, we could not analyze the role of age, gender, lifestyle, and other genetic variations, on the adjusted association of VDR gene SNPs and urolithiasis risk. Hence, additional works with respect to the gene-gene and gene-environment interactions is needed to approach with a more comprehensive estimation. Third, we noticed a significant heterogeneity among the studies for various comparisons, which may impress the perception of findings. Although we conducted subgroup analysis and weighted meta-regression in order to attenuate its effects. Finally, there were a number of VDR gene SNPs in the context of urolithiasis risk that could not be included in the meta-analysis due to lack of sufficient amount of data. Hence, it could barely implied that $V D R$ gene could not convey a genetic risk factor for urolithiasis, merely regarding our findings.

\section{Conclusion}

In conclusion, the results of pooled analysis did not demonstrate any statistically significant association between all four SNPs and susceptibility to urolithiasis. However, subgroup analysis showed that the 
Recessive, allelic, and aa vs. AA model of ApaI and Tt vs. TT comparison of the TaqI gene polymorphism increased risk of urolithiasis in East-Asian and Caucasians population, respectively. Further genes should be evaluated to disclose the genetic mechanisms contributing to urolithiasis development. Moreover, the role of life style, age, and gender needs be considered in the stratification analyses for $V D R$ gene SNPs and urolithiasis predisposition.

\section{Abbreviations}

VD: Vitamin D receptor; Cl: Confidence interval; OR: Odds ratio; SNP: Singlenucleotide polymorphism; CaSR: Calcium sensing receptor;

OPN: Osteopontin; PAQR6: Progestin and adipo receptor 6; PRISMA: Preferred Reporting Items for Systematic reviews and Meta-Analyses; NOS: NewcastleOttawa scale; HWE: Hardy-Weinberg equilibrium; RXR: Retinoid X receptor; VDRE: Vitamin D response element

\section{Acknowledgements}

The authors would like to thank Mrs. Zahra Hosseinzadeh for all her support.

\section{Authors' contributions}

$\mathrm{DI}$ and RR originated the study and acquired data. BR, KL, and AK performed statistical analysis, interpreted data, and drafted the manuscript. SA, MM, and AK revised the manuscript. All authors read and approved the final manuscript.

\section{Funding}

Not applicable.

\section{Availability of data and materials}

Not applicable.

Ethics approval and consent to participate

Not applicable.

\section{Consent for publication}

Not applicable.

\section{Competing interests}

The authors declare that they have no conflict of interest to report.

\section{Author details}

${ }^{1}$ Department of Immunology, School of Public Health, Tehran University of Medical Sciences, Tehran, Iran. ${ }^{2}$ Department of Hematology, School of Medicine, Tarbiat Modares University, Tehran, Iran. ${ }^{3}$ Department of Medical Immunology, Faculty of Medical Sciences, Tarbiat Modares University, Tehran, Iran. ${ }^{4}$ Department of Physiology, School of Medicine, Tehran University of Medical Sciences, Tehran, Iran. ${ }^{5}$ Department of Immunology, Faculty of Medicine, Iran University of Medical Sciences, Tehran, Iran. ${ }^{6}$ Immunology Research Center, Iran University of Medical Sciences, Tehran, Iran. ${ }^{7}$ Department of Immunology, Medical School, Shahid Beheshti University of Medical Sciences, Tehran, Iran. ${ }^{8}$ Department of Immunology, School of Medicine, Tehran University of Medical Sciences, Tehran, Iran.

Received: 6 November 2019 Accepted: 1 July 2020

Published online: 10 July 2020

\section{References}

1. Heyns C. Urinary tract infection associated with conditions causing urinary tract obstruction and stasis, excluding urolithiasis and neuropathic bladder. World J Urol. 2012;30(1):77-83.

2. Lee $Y-H$, Huang W-C, Tsai J-Y, Lu C-M, Chen W-C, Lee M-H, et al. Epidemiological studies on the prevalence of upper urinary calculi in Taiwan. Urol Int. 2002;68(3):172-7.

3. Ngo TC, Assimos DG. Uric acid nephrolithiasis: recent progress and future directions. Rev Urol. 2007;9(1):17.
4. Gao B, Yasui T, Okada A, Tozawa K, Hayashi Y, Kohri K. A polymorphism of the osteopontin gene is related to urinary calcium stones. J Urol. 2005;174(4 Part 1):1472-6.

5. Hamilton D, Grover V, Smith C, Cole D. Heterogeneous disease modeling for hardy-Weinberg disequilibrium in case-control studies: application to renal stones and calcium-sensing receptor polymorphisms. Ann Hum Genet. 2009;73(2):176-83.

6. Rungroj N, Nettuwakul C, Sudtachat N, Praditsap O, Sawasdee N, Sritippayawan S, et al. A whole genome SNP genotyping by DNA microarray and candidate gene association study for kidney stone disease. BMC Med Genet. 2014;15(1):50.

7. Zhu C, Ye Z, Chen Z, Xia D, Hu J. Association between vitamin D receptor gene polymorphisms and idiopathic hypocitraturia in the Chinese population. Urol Int. 2010;85(1):100-5.

8. Thorleifsson G, Holm H, Edvardsson V, Walters GB, Styrkarsdottir U, Gudbjartsson DF, et al. Sequence variants in the CLDN14 gene associate with kidney stones and bone mineral density. Nat Genet. 2009;41(8):926.

9. Urabe Y, Tanikawa C, Takahashi A, Okada Y, Morizono T, Tsunoda T, et al. A genome-wide association study of nephrolithiasis in the Japanese population identifies novel susceptible Loci at 5q35. 3, 7p14. 3, and 13q14. 1. PLoS Genet. 2012;8(3):e1002541.

10. Vezzoli G, Terranegra A, Arcidiacono T, Soldati L. Genetics and calcium nephrolithiasis. Kidney Int. 2011;80(6):587-93.

11. Knoll T. Epidemiology, pathogenesis, and pathophysiology of urolithiasis. Eur Urol Suppl. 2010;9(12):802-6.

12. Khan SR, Byer KJ, Thamilselvan S, Hackett RL, Mccormack WT, Benson NA, et al. Crystal-cell interaction and apoptosis in oxalate-associated injury of renal epithelial cells. J Am Soc Nephrol. 1999;10:S457-63.

13. Kok D. Crystallization and stone formation inside the nephron. Scanning Microsc. 1996;10(2):471-84 discussion 84-6.

14. Evan AP, Lingeman JE, Coe FL, Parks JH, Bledsoe SB, Shao Y, et al. Randall's plaque of patients with nephrolithiasis begins in basement membranes of thin loops of Henle. J Clin Invest. 2003;111(5):607-16.

15. de Water R, Noordermeer C, Houtsmuller AB, Nigg AL, Stijnen T, Schröder $\mathrm{FH}$, et al. Role of macrophages in nephrolithiasis in rats: an analysis of the renal interstitium. Am J Kidney Dis. 2000;36(3):615-25.

16. Letavernier $\mathrm{E}$, Daudon M. Vitamin D, hypercalciuria and kidney stones. Nutrients. 2018;10(3):366.

17. Parivar F, Low RK, Stoller ML. The influence of diet on urinary stone disease. J Urol. 1996;155(2):432-40.

18. Gocek E, Kiełbiński M, Marcinkowska E. Activation of intracellular signaling pathways is necessary for an increase in VDR expression and its nuclear translocation. FEBS Lett. 2007:581(9):1751-7.

19. Mittal RD, Bid HK, Manchanda PK, Kapoor R. Predisposition of genetic polymorphism with the risk of urolithiasis. Indian J Clin Biochem. 2008;23(2): 106-16.

20. Nejentsev S, Godfrey L, Snook H, Rance H, Nutland S, Walker NM, et al. Comparative high-resolution analysis of linkage disequilibrium and tag single nucleotide polymorphisms between populations in the vitamin D receptor gene. Hum Mol Genet. 2004;13(15):1633-9.

21. Zhou T-B, Jiang Z-P, Huang M-F, Su N. Association of vitamin D receptor Fok1 (rs2228570), Taql (rs731236) and Apal (rs7975232) gene polymorphism with the risk of chronic kidney disease. J Recept Signal Transduct Res. 2015; 35(1):58-62.

22. Imani D, Razi B, Motallebnezhad M, Rezaei R. Association between vitamin D receptor (VDR) polymorphisms and the risk of multiple sclerosis (MS): an updated meta-analysis. BMC Neurol. 2019;19(1):339.

23. Makoui MH, Imani D, Motallebnezhad M, Azimi M, Razi B. Vitamin D receptor gene polymorphism and susceptibility to asthma: meta-analysis based on 17 case-control studies. Ann Allergy Asthma Immunol. 2020; 124(1):57-69.

24. Jackman SV, Kibel AS, Ovuworie CA, Moore RG, Kavoussi LR, Jarrett TW. Familial calcium stone disease: Taq I polymorphism and the vitamin D receptor. J Endourol. 1999;13(4):313-6.

25. Ruggiero $M$, Pacini S, Amato $M$, Aterini S, Chiarugi V. Association between vitamin D receptor gene polymorphism and nephrolithiasis. Miner Electrolyte Metab. 1999;25(3):185-90.

26. Chen W-C, Chen H-Y, Hsu C-D, Wu J-Y, Tsai F-J. No association of vitamin D receptor gene Bsm I polymorphisms with calcium oxalate stone formation. Mol Urol. 2001;5(1):7-10. 
27. Chen WC, Chen HY, Lu HF, Hsu CD, Tsai FJ. Association of the vitamin D receptor gene start codon Fok I polymorphism with calcium oxalate stone disease. BJU Int. 2001:87(3):168-71.

28. Nishijima S, Sugaya K, Naito A, Morozumi M, Hatano T, Ogawa Y. Association of vitamin D receptor gene polymorphism with urolithiasis. J Urol. 2002;167(5):2188-91.

29. Özkaya O, Söylemezoğlu O, Mısırlığlu M, Gönen S, Buyan N, Hasanoğlu E. Polymorphisms in the vitamin D receptor gene and the risk of calcium nephrolithiasis in children. Eur Urol. 2003;44(1):150-4.

30. Shaogang $W$, Jihong $L$, Shaoqun $H$, Zhangqun $Y$. Association of vitamin $\mathrm{D}$ receptor gene polymorphisms with calcium oxalate calculus disease. J Huazhong Univ Sci Technol [Medical Sciences]. 2003;23(1):38-41.

31. Mossetti G, Rendina D, Viceconti R, Manno G, Guadagno V, Strazzullo P, et al. The relationship of $3^{\prime}$ vitamin $D$ receptor haplotypes to urinary supersaturation of calcium oxalate salts and to age at onset and familial prevalence of nephrolithiasis. Nephrol Dial Transplant. 2004; 19(9):2259-65.

32. Relan V, Khullar M, Singh S, Sharma S. Association of vitamin D receptor genotypes with calcium excretion in nephrolithiatic subjects in northern India. Urol Res. 2004;32(3):236-40.

33. Rendina D, Mossetti G, Viceconti R, Sorrentino M, Castaldo R, Manno G, et al. Association between vitamin $D$ receptor gene polymorphisms and fasting idiopathic hypercalciuria in recurrent stone-forming patients. Urology. 2004; 64(4):833-8.

34. Bid HK, Chaudhary H, Mittal RD. Association of vitamin-D and calcitonin receptor gene polymorphism in paediatric nephrolithiasis. Pediatr Nephrol. 2005;20(6):773-6.

35. Bid HK, Kumar A, Kapoor R, Mittal RD. Association of vitamin D receptorgene (Fokl) polymorphism with calcium oxalate nephrolithiasis. J Endourol. 2005:19(1):111-5.

36. Gunes S, Bilen CY, Kara N, Asci R, Bagci H, Yilmaz AF. Vitamin D receptor gene polymorphisms in patients with urolithiasis. Urol Res. 2006;34(1):47-52.

37. Liu CC, Huang CH, Wu WJ, Huang SP, Chou YH, Li CC, et al. Association of vitamin $D$ receptor (Fok-I) polymorphism with the clinical presentation of calcium urolithiasis. BJU Int. 2007;99(6):1534-8.

38. Franco MJM, de Tejada Romero MJG, Lozano RG, Moruno R, Ortega R, Martí $V$, et al. Changes in bone mineral metabolism in patients with recurrent urolithiasis and vitamin D receptor gene polymorphisms. Preliminary results. Nefrología (English Edition). 2007;27(6):694-703.

39. Seyhan S, Yavascaoglu I, Kilicarslan H, Dogan HS, Kordan Y. Association of vitamin D receptor gene Taq I polymorphism with recurrent urolithiasis in children. Int J Urol. 2007;14(12):1060-2.

40. Seo IY, Kang I-H, Chae S-C, Park SC, Lee Y-J, Yang YS, et al. Vitamin D receptor gene Alw I, Fok I, Apa I, and Taq I polymorphisms in patients with urinary stone. Urology. 2010;75(4):923-7.

41. Mittal RD, Mishra DK, Srivastava P, Manchanda P, Bid H, Kapoor R. Polymorphisms in the vitamin $\mathrm{D}$ receptor and the androgen receptor gene associated with the risk of urolithiasis. Indian J Clin Biochem. 2010;25(2): 119-26.

42. Basiri A, Shakhssalim N, Houshmand M, Kashi AH, Azadvari M, Golestan $B$, et al. Coding region analysis of vitamin $D$ receptor gene and its association with active calcium stone disease. Urol Res. 2012;40(1):35-40.

43. Aji K, Song GL, Yasen A, Azad B, Tursun H. Association of vitamin Dreceptor gene polymorphism with urolithiasis in Uyghur children from southern Xinjiang,China. Chine J Contempt Pediatr. 2012;2:956-9.

44. Wang $\mathrm{S}$, Wang $X$, Wu J, Lin $Y$, Chen $H$, Zheng $X$, et al. Association of vitamin $D$ receptor gene polymorphism and calcium urolithiasis in the Chinese Han population. Urol Res. 2012;40(4):277-84.

45. Aykan S, Tuken M, Gunes S, Akin Y, Ozturk M, Seyhan S, et al. ApaL1 urokinase and Taq1 vitamin D receptor gene polymorphisms in first-stone formers, recurrent stone formers, and controls in a Caucasian population. Urolithiasis. 2016;44(2):109-15.

46. Goknar N, Öktem F, Torun E, Gok O, Demir AD, Kucukkoc M, et al. The role of vitamin $D$ receptor gene polymorphisms in Turkish infants with urolithiasis. Ren Fail. 2016;38(4):545-51.

47. Ergon EY, Akil IO, Taneli F, Oran A, Ozyurt BC. Etiologic risk factors and vitamin $D$ receptor gene polymorphisms in under one-year-old infants with urolithiasis. Urolithiasis. 2017:1-8.

48. Subaşı B, Gökçe I, Delil K, Alpay H. Vitamin D receptor gene polymorphisms in children with kidney stone disease. Turk J Pediatr. 2017;59(4):404-9.
49. Li K, Luo Y, Mo Y, Shen J, Liu X, Li H. Association between vitamin D receptor gene polymorphisms and idiopathic hypocitraturia in a Chinese Bai population. Urolithiasis. 2018:1-8.

50. Huang Y, Peng Q, Bao M, Liu C, Wu K, Zhou S. Biochemical metabolic levels and vitamin D receptor Fokl gene polymorphisms in Uyghur children with urolithiasis. PLoS One. 2019;14(2):e0212183.

51. Lin Y, Mao Q, Zheng X, Chen H, Yang K, Xie L. Vitamin D receptor genetic polymorphisms and the risk of urolithiasis: a meta-analysis. Urol Int. 2011; 86(3):249-55.

52. Zhang $P$, Nie $W$, Jiang $H$. Effects of vitamin $D$ receptor polymorphisms on urolithiasis risk: a meta-analysis. BMC Med Genet. 2013;14(1):104.

53. Liu W, Chen M, Li M, Ma H, Tong S, Lei Y, et al. Vitamin D receptor gene (VDR) polymorphisms and the urolithiasis risk: an updated meta-analysis based on 20 case-control studies. Urolithiasis. 2014;42(1):45-52.

54. Moher D, Liberati A, Tetzlaff J, Altman DG. Preferred reporting items for systematic reviews and meta-analyses: the PRISMA statement. Ann Intern Med. 2009;151(4):264-9.

55. Zeng X, Zhang Y, Kwong JS, Zhang C, Li S, Sun F, et al. The methodological quality assessment tools for preclinical and clinical studies, systematic review and meta-analysis, and clinical practice guideline: a systematic review. J Evid Based Med. 2015;8(1):2-10.

56. Rodriguez S, Gaunt TR, Day IN. Hardy-Weinberg equilibrium testing of biological ascertainment for Mendelian randomization studies. Am J Epidemiol. 2009;169(4):505-14.

57. DerSimonian R, Laird N. Meta-analysis in clinical trials revisited. Contemporary clinical trials. 2015:45:139-45.

58. Mantel N, Haenszel W. Statistical aspects of the analysis of data from retrospective studies of disease. J Natl Cancer Inst. 1959;22(4):719-48.

59. Egger M, Smith GD, Schneider M, Minder C. Bias in meta-analysis detected by a simple, graphical test. Bmj. 1997;315(7109):629-34.

60. Murray KE, Moore L, O'Brien C, Clohessy A, Brophy C, Minnock P, et al. Updated pharmacological management of rheumatoid arthritis for women before, during, and after pregnancy, reflecting recent guidelines. Ir J Med Sci (1971-). 2019;188(1):169-72.

61. Aji K, Song G, Yasen A, Azad B, Tursun H. Association of vitamin D receptor gene polymorphisms with urolithiasis in Uyghur children from southern Xinjiang, China. Zhongguo Dang Dai Er Ke Za Zhi. 2012;14(12):956-9.

62. Amar A, Afzal A, Hussain SA, Hameed A, Khan AR, Shakoor M, et al. Association of vitamin D receptor gene polymorphisms and risk of urolithiasis: results of a genetic epidemiology study and comprehensive meta-analysis. Urolithiasis. 2019:1-17.

63. Guha M, Bankura B, Ghosh S, Pattanayak AK, Ghosh S, Pal DK, et al. Polymorphisms in CaSR and CLDN14 genes associated with increased risk of kidney stone disease in patients from the eastern part of India. PloS one. 2015;10(6).

64. Ergon EY, Akil iO, Taneli F, Oran A, Ozyurt BC. Etiologic risk factors and vitamin $D$ receptor gene polymorphisms in under one-year-old infants with urolithiasis. Urolithiasis. 2018;46(4):349-56.

65. Cakir OO, Yilmaz A, Demir E, Incekara K, Kose MO, Tunali NE. Association of the Bsml, Apal, Taql, Tru9l and Fokl polymorphisms of the vitamin D receptor gene with nephrolithiasis in the Turkish population. Urol J. 2016; 13(1):2509-18.

66. Yang Z, Wang Q, Zhong JF, Li L. Polymorphisms of the VDR gene in patients with nephrolithiasis in a Han Chinese population. Urolithiasis. 2019; 47(2):149-54.

67. Mossetti G, Vuotto P, Rendina D, Numis F, Viceconti R, Giordano F, et al. Association between vitamin $D$ receptor gene polymorphisms and tubular citrate handling in calcium nephrolithiasis. J Intern Med. 2003; 253(2):194-200

68. Li K, Luo Y, Mo Y, Shen J, Liu X, Li H. Association between vitamin D receptor gene polymorphisms and idiopathic hypocitraturia in a Chinese Bai population. Urolithiasis. 2019;47(3):235-42.

69. Vanessa O, Asani FF, Jeffery TJ, Saccone DS, Bornman L. Vitamin D receptor gene expression and function in a south African population: ethnicity, vitamin D and Fokl. PLoS One. 2013;8(6):e67663.

70. Oleson CV, Patel PH, Wuermser L-A. Influence of season, ethnicity, and chronicity on vitamin D deficiency in traumatic spinal cord injury. J Spinal Cord Med. 2010;33(3):202-13.

71. Chawla D, Daniels JL, Benjamin-Neelon SE, Fuemmeler BF, Hoyo C, Buckley JP. Racial and ethnic differences in predictors of vitamin D among pregnant women in South-Eastern USA. J Nutr Sci. 2019;8. 
72. Dickinson JL, Perera DI, Van der Mei A, Ponsonby A-L, Polanowski AM, Thomson RJ, et al. Past environmental sun exposure and risk of multiple sclerosis: a role for the $\mathrm{Cdx}-2$ vitamin $\mathrm{D}$ receptor variant in this interaction. Mult Scler J. 2009;15(5):563-70.

73. Correia A, MdS A, Gondim F, Bandeira F. Ethnic aspects of vitamin D deficiency. Arq Bras Endocrinol Metabol. 2014;58(5):540-4.

74. Li F, Jiang L, Willis-Owen SA, Zhang Y, Gao J. Vitamin D binding protein variants associate with asthma susceptibility in the Chinese Han population. BMC Med Genet. 2011;12(1):103.

75. Fang W-L, Gao L-B, Liang W-B, Xue H, Bai P, Lv M-L, et al. Association analysis of vitamin $D$ receptor gene polymorphisms in chinese population with asthma. Iran J Allergy Asthma Immunol. 2009:141-7.

76. Ismail MF, Elnady HG, Fouda EM. Genetic variants in vitamin D pathway in Egyptian asthmatic children: a pilot study. Hum Immunol. 2013; 74(12):1659-64.

77. Pillai DK, labal SF, Benton AS, Lerner J, Wiles A, Foerster M, et al. Associations between genetic variants in vitamin D metabolism and asthma characteristics in young African Americans: a pilot study. J Investig Med. 2011;59(6):938-46

78. González-Castro TB, Blachman-Braun R, Hernández-Díaz Y, Tovilla-Zárate CA, Pérez-Hernández N, Moscardi PRM, et al. Association of vitamin D receptor polymorphisms and nephrolithiasis: a meta-analysis. Gene. 2019;711:143936.

79. Holick MF. McCollum award lecture, 1994: vitamin D-new horizons for the 21st century. Oxford: Oxford University Press; 1994

80. Roy C, Gies H, Toomey S. The solar UV radiation environment: measurement techniques and results. J Photochem Photobiol B Biol. 1995;31(1-2):21-7.

81. Purdue MP, Hartge P, Davis S, Cerhan JR, Colt JS, Cozen W, et al. Sun exposure, vitamin D receptor gene polymorphisms and risk of non-Hodgkin lymphoma. Cancer Causes Control. 2007;18(9):989-99.

82. Medeiros R, Morais A, Vasconcelos A, Costa S, Pinto D, Oliveira J, et al. The role of vitamin $D$ receptor gene polymorphisms in the susceptibility to prostate cancer of a southern European population. J Hum Genet. 2002; 47(8):413-8.

83. Bodiwala D, Luscombe CJ, French ME, Liu S, Saxby MF, Jones PW, et al. Polymorphisms in the vitamin D receptor gene, ultraviolet radiation, and susceptibility to prostate cancer. Environ Mol Mutagen. 2004;43(2):121-7.

\section{Publisher's Note}

Springer Nature remains neutral with regard to jurisdictional claims in published maps and institutional affiliations.

Ready to submit your research? Choose BMC and benefit from:

- fast, convenient online submission

- thorough peer review by experienced researchers in your field

- rapid publication on acceptance

- support for research data, including large and complex data types

- gold Open Access which fosters wider collaboration and increased citations

- maximum visibility for your research: over $100 \mathrm{M}$ website views per year

At $\mathrm{BMC}$, research is always in progress.

Learn more biomedcentral.com/submissions 